\title{
Pseudo Kobayashi hyperbolicity of subvarieties of general type on abelian varieties
}

\author{
By Katsutoshi YamanoI
}

(Received Aug. 10, 2016)

(Revised Aug. 17, 2017)

\begin{abstract}
We prove that the Kobayashi pseudo distance of a closed subvariety $X$ of an abelian variety $A$ is a true distance outside the special set $\operatorname{Sp}(X)$ of $X$, where $\operatorname{Sp}(X)$ is the union of all positive dimensional translated abelian subvarieties of $A$ which are contained in $X$. More strongly, we prove that a closed subvariety $X$ of an abelian variety is taut modulo $\operatorname{Sp}(X)$; Every sequence $f_{n}: \mathbb{D} \rightarrow X$ of holomorphic mappings from the unit disc $\mathbb{D}$ admits a subsequence which converges locally uniformly, unless the image $f_{n}(K)$ of a fixed compact set $K$ of $\mathbb{D}$ eventually gets arbitrarily close to $\operatorname{Sp}(X)$ as $n$ gets larger. These generalize a classical theorem on algebraic degeneracy of entire curves in irregular varieties.
\end{abstract}

\section{Introduction.}

Let $X$ be a complex space. In this paper, by complex space, we mean a reduced and irreducible complex space, unless otherwise specified. Let $\Delta \subset X$ be a closed subset. We say that $X$ is Kobayashi hyperbolic modulo $\Delta$ if the Kobayashi pseudo distance $d_{X}$ of $X$ satisfies $d_{X}(p, q)>0$ for every pair of distinct points $p, q \in X$ not both contained in $\Delta$. When $\Delta$ is an empty set, this definition reduces to the usual definition of Kobayashi hyperbolicity. We say that a complex projective variety $X$ is pseudo Kobayashi hyperbolic if there exists a proper Zariski closed subset $Z \varsubsetneqq X$ such that $X$ is Kobayashi hyperbolic modulo $Z$. It is conjectured that a projective variety $X$ is pseudo Kobayashi hyperbolic if $X$ is of general type (cf. [19, 7.4.13], [21, p.180]). We discuss this problem when $X$ is a closed subvariety of an abelian variety. We first state a corollary of our main theorem.

Corollary 1.1. Let $X$ be a closed subvariety of an abelian variety. Assume that $X$ is of general type. Then $X$ is pseudo Kobayashi hyperbolic.

To make the statement more precise, we introduce the special set. Let $X$ be a closed subvariety of an abelian variety $A$. We define the special set $\operatorname{Sp}(X)$ of $X$ by

$$
\operatorname{Sp}(X)=\{x \in X ; \exists B \subset A, \text { an abelian variety s.t. } \operatorname{dim}(B)>0 \text { and } x+B \subset X\} .
$$

Then fundamental facts due to Ueno [32, Theorem 10.9] and Kawamata [15, Theorem 4] are the followings:

2010 Mathematics Subject Classification. Primary 32Q45; Secondary 32H30, 14K12.

Key Words and Phrases. pseudo Kobayashi hyperbolicity, tautness, Nevanlinna theory.

The author was supported by JSPS Grant-in-Aid for Scientific Research (C), 24540069 and by JSPS Grant-in-Aid for Scientific Research (B), 17H02842. 
- (Ueno) If $X$ is not of general type, then $\operatorname{Sp}(X)=X$.

- (Kawamata) If $X$ is of general type, then $\operatorname{Sp}(X)$ is a Zariski closed subset of $X$ and $\operatorname{Sp}(X) \neq X$.

We remark that $\operatorname{Sp}(X)$ is an empty set if $X$ is Kobayashi hyperbolic. This follows from the vanishing $d_{B} \equiv 0$ of the Kobayashi pseudo distances $d_{B}$ of abelian varieties $B$ and the distance decreasing property of Kobayashi pseudo distances. We refer the readers to $[\mathbf{2 1}$, p.36] and $[\mathbf{7}$, p.119] for another, but equivalent definition of the special set, which works for general projective varieties.

We state our main theorem.

TheOREM 1.2. Let $X$ be a closed subvariety of an abelian variety. Then $X$ is Kobayashi hyperbolic modulo $\operatorname{Sp}(X)$.

By Kawamata's theorem above, Corollary 1.1 immediately follows from Theorem 1.2. By Ueno's theorem above, Theorem 1.2 is trivial when $X$ is not of general type, because $X$ is always Kobayashi hyperbolic modulo $X$ itself. We may generalize our theorem to the case of closed complex subspaces of complex tori (cf. Corollary 11.3). However, this generalization is not essential, because a closed complex subspace $X$ of a complex torus is actually a closed subvariety of an abelian variety, provided that $X$ is of general type (cf. Lemma 11.1).

We remark that $\operatorname{Sp}(X)$ is the minimum closed subset of $X$ that can be taken in the statement of Theorem 1.2; If a closed subvariety $X$ of an abelian variety is Kobayashi hyperbolic modulo some closed subset $\Delta \subset X$, then $\Delta$ necessarily contains $\operatorname{Sp}(X)$. Indeed, suppose $x \in \operatorname{Sp}(X)$. We take a positive dimensional abelian variety $B \subset A$ such that $x+B \subset X$, and a point $b \in B$ with $b \neq 0$. By $d_{B} \equiv 0$, the distance decreasing property yields $d_{X}(x, x+b)=0$. This shows $x \in \Delta$ (and $x+b \in \Delta$ ), and hence $\operatorname{Sp}(X) \subset \Delta$.

As a consequence of this observation, we get a converse of Corollary 1.1.

COROLlary 1.3. A closed subvariety of an abelian variety is of general type if and only if it is pseudo Kobayashi hyperbolic.

Indeed, if a closed subvariety $X$ of an abelian variety is not of general type, then $\operatorname{Sp}(X)=X$ by Ueno's theorem above. Hence $X$ is not Kobayashi hyperbolic modulo $Z$ for any proper Zariski closed subset $Z \varsubsetneqq X$ because of the minimality of $\operatorname{Sp}(X)$. Thus $X$ is not pseudo Kobayashi hyperbolic.

Theorem 1.2 is a generalization of a theorem of Green [9], who proved that a closed subvariety $X$ of an abelian variety is Kobayashi hyperbolic, if $\operatorname{Sp}(X)$ is empty. His proof is based on Brody's criterion of compact Kobayashi hyperbolic spaces: A compact complex space $V$ is Kobayashi hyperbolic if there is no entire curve $\mathbb{C} \rightarrow V$ with bounded derivatives. Here an entire curve $\mathbb{C} \rightarrow V$ is a non-constant holomorphic map from the complex plane $\mathbb{C}$. Thus the proof is reduced to show the non-existence of an entire curve $\mathbb{C} \rightarrow X$ with bounded derivatives under the assumption $\operatorname{Sp}(X)=\emptyset$. However unlike the case of Kobayashi hyperbolicity, no criterion is known for pseudo Kobayashi hyperbolicity in terms of entire curves. This is a major difference between the problem of Kobayashi hyperbolicity and that of pseudo Kobayashi hyperbolicity (cf. [34]). 
Our proof of Theorem 1.2 is based on a stronger result. To state it, we introduce another terminology called tautness (cf. [19, Chapter 5]). Let $X$ be a complex space. Let $\operatorname{Hol}(\mathbb{D}, X)$ be the set of all holomorphic mappings from the unit disc $\mathbb{D}=\{z \in \mathbb{C} ;|z|<1\}$ to $X$. Let $\Delta \subset X$ be a closed subset. We say that $X$ is taut modulo $\Delta$ if for each sequence $\left\{f_{n}\right\}_{n=1}^{\infty}$ in $\operatorname{Hol}(\mathbb{D}, X)$, we have one of the followings: (1) $\left\{f_{n}\right\}_{n=1}^{\infty}$ has a subsequence which converges locally uniformly to some $f \in \operatorname{Hol}(\mathbb{D}, X)$, or $(2)$ for each compact subset $K \subset \mathbb{D}$ and each compact subset $L \subset X \backslash \Delta$, there exists an integer $n_{0}$ such that $f_{n}(K) \cap L=\emptyset$ for all $n \geq n_{0}$. We have the following theorem of Kiernan and Kobayashi (cf. [16, Theorem 1], $[\mathbf{1 9}, 5.1 .3])$ : If $X$ is taut modulo $\Delta$, then $X$ is Kobayashi hyperbolic modulo $\Delta$. (We remark that the converse of this does not hold. See Example 2.2.) Hence Theorem 1.2 immediately follows from the following theorem.

TheOrem 1.4. Let $X$ be a closed subvariety of an abelian variety. Then $X$ is taut modulo $\operatorname{Sp}(X)$.

According to a geometric interpretation due to [16], a variant of Theorem 1.4 for a closed subvariety of an algebraic torus, instead of an abelian variety, includes a classical theorem of Bloch [1] , supplemented by Cartan [5] (cf. [20, Chapter VIII]). This will be achieved by generalizing Theorem 1.4 to the logarithmic case, namely the case of subvarieties of semi-abelian varieties.

As an application of Corollary 1.1, we prove a generalization of the theorem of BlochOchiai. For a compact complex manifold $X$, the irregularity $q(X)$ of $X$ is defined by $q(X)=\operatorname{dim} H^{0}\left(X, \Omega_{X}^{1}\right)$. The statement of the Bloch-Ochiai theorem is the following: If $X$ is a projective manifold such that $q(X)>\operatorname{dim}(X)$, then no entire curve $f: \mathbb{C} \rightarrow X$ has Zariski dense image in $X$. This statement was first claimed by Bloch [2] with incomplete proof in 1920's. Then it was proved by Ochiai [29] and Kawamata [15]. A generalization to the logarithmic case was proved by Noguchi $[\mathbf{2 5}]$. See also $[\mathbf{1 0}],[\mathbf{1 7}],[\mathbf{2 2}],[\mathbf{2 7}]$. For the detailed discussion of this theorem including its history, we refer the readers to $[\mathbf{2 8}$, Section 4.8]. We generalize the Bloch-Ochiai theorem as follows.

Corollary 1.5. Let $X$ be a compact Kähler manifold such that $q(X)>\operatorname{dim} X$. Then for every $x \in X$, the set $E_{x}=\left\{y \in X ; d_{X}(x, y)=0\right\}$ is contained in a proper analytic subset of $X$.

The implication of the Bloch-Ochiai theorem from Corollary 1.5 is as follows. Let $f: \mathbb{C} \rightarrow X$ be an entire curve into a projective manifold $X$ with $q(X)>\operatorname{dim} X$. We have $d_{X}(f(0), f(z))=0$ for all $z \in \mathbb{C}$. This follows from the vanishing $d_{\mathbb{C}} \equiv 0$ of Kobayashi pseudo distance $d_{\mathbb{C}}$ of the complex plane $\mathbb{C}$. Hence $f(\mathbb{C}) \subset E_{f(0)}$. Thus by Corollary 1.5, $f(\mathbb{C})$ is contained in a proper analytic (hence algebraic) subset of $X$.

The outline of this paper is as follows. In Section 2, we derive Theorem 1.4 from a Schottky-Landau type estimate (cf. Theorem 2.1) for holomorphic maps $f: \mathbb{D} \rightarrow X$ from the unit disc $\mathbb{D}$ to a closed subvariety $X$ of an abelian variety.

The most of the paper (Sections 3 to 10) is devoted to the proof of Theorem 2.1. An important issue in the proof of Theorem 2.1 is to formulate an appropriate proposition (cf. Proposition 2.3), from which we can derive Theorem 2.1, and for which we can adapt induction. In Sections 3-8, we prove Proposition 2.3. More detailed outline of the proof 
of Proposition 2.3 is discussed at the end of Section 2. In Sections 9 and 10, we derive Theorem 2.1 from Proposition 2.3.

In Section 11, we generalize our results to the case of complex subspaces of complex tori, and prove Corollary 1.5.

REMARK 1.6. In this paper, an algebraic variety (or simply a variety) is an integral, separated scheme of finite type over the complex number field $\mathbb{C}$ (cf., e.g., [12, p.105]). In particular, every variety is reduced, irreducible and non-empty (cf. [12, Chapter II, Proposition 3.1]). Every variety has a canonically associated complex space structure (cf. [12, p.439], [30, Section 2]).

\section{A Schottky-Landau type estimate.}

Let $M$ be a complex manifold, and let $\omega_{M}$ be a smooth, positive $(1,1)$-form on $M$. For $v \in T M$, we denote by $|v|_{\omega_{M}}$ the norm of $v$ defined by the Hermitian metric on $M$ associated to $\omega_{M}$. When $A$ is an abelian variety, we equip $A$ with a positive $(1,1)$-form $\omega_{A}$ which is invariant under the translation of $A$. We call this $\omega_{A}$ a positive invariant $(1,1)$-form. A positive invariant $(1,1)$-form $\omega_{A}$ is expressed as

$$
\omega_{A}=\frac{i}{2}\left(d z_{1} \wedge d \bar{z}_{1}+\cdots+d z_{n} \wedge d \bar{z}_{n}\right)
$$

for some basis $d z_{1}, \ldots, d z_{n} \in \Gamma\left(A, \Omega_{A}^{1}\right)$, where $n=\operatorname{dim} A$. A crucial consequence of this presentation (2.1) is that, for every holomorphic map $f: \mathbb{D} \rightarrow A$, the function $\log \left|f^{\prime}(z)\right|_{\omega_{A}}$ is subharmonic on $\mathbb{D}$. For $s>0$, we set $\mathbb{D}(s)=\{z \in \mathbb{C} ;|z|<s\}$ : hence, $\mathbb{D}(1)=\mathbb{D}$.

TheOrem 2.1. Let $X$ be a closed subvariety of an abelian variety A. Assume that $X$ is of general type. Let $\omega_{A}$ be a positive invariant $(1,1)$-form on $A$. Let $U \subset X$ be an open neighborhood of $\operatorname{Sp}(X)$ and let $0<s<1$. Then there exists a positive constant $c>0$ such that for every $f \in \operatorname{Hol}(\mathbb{D}, X)$ with $f(\mathbb{D}(s)) \not \subset U$, we have $\left|f^{\prime}(0)\right|_{\omega_{A}} \leq c$.

Derivation of Theorem 1.4 From Theorem 2.1. We remark that Theorem 1.4 is obvious if $X$ is not of general type. Indeed, in this case, $\operatorname{Sp}(X)=X$, but $X$ is always taut modulo $X$ itself. Hence in the following, we assume that $X$ is of general type.

Let $\left\{f_{n}\right\}$ be a sequence in $\operatorname{Hol}(\mathbb{D}, X)$. Assume that there exist a compact set $K \subset \mathbb{D}$ and an open set $U \subset X$ with $\operatorname{Sp}(X) \subset U$ such that $f_{n}(K) \not \subset U$ for arbitrarily large $n$. By taking a subsequence if necessary, we may assume that $f_{n}(K) \not \subset U$ for all $n$. Let $0<\sigma<1$. We shall show that the sequence $\left\{f_{n}\right\}$ is equi-continuous on $\mathbb{D}(\sigma)$. For each $w \in \mathbb{D}(\sigma)$, let $Q_{w}: \mathbb{D} \rightarrow \mathbb{D}$ be a conformal automorphism such that $Q_{w}(0)=w$. Let $s \in(0,1)$ be a constant such that $Q_{w}^{-1}(K) \subset \mathbb{D}(s)$ for all $w \in \mathbb{D}(\sigma)$. Then

$$
f_{n} \circ Q_{w}(\mathbb{D}(s)) \not \subset U
$$

for all $n$ and all $w \in \mathbb{D}(\sigma)$. Hence by Theorem 2.1, there exists a positive constant $c>0$ such that 


$$
\left|\left(f_{n} \circ Q_{w}\right)^{\prime}(0)\right|_{\omega_{A}} \leq c
$$

for all $n$ and all $w \in \mathbb{D}(\sigma)$. Hence

$$
\left|f_{n}^{\prime}(w)\right|_{\omega_{A}}=\frac{\left|\left(f_{n} \circ Q_{w}\right)^{\prime}(0)\right|_{\omega_{A}}}{\left|Q_{w}^{\prime}(0)\right|} \leq \frac{c}{1-\sigma^{2}}
$$

for all $n$ and all $w \in \mathbb{D}(\sigma)$. This shows that the sequence $\left\{f_{n}\right\}$ is equi-continuous on $\mathbb{D}(\sigma)$. By the Arzelà-Ascoli theorem, the sequence $\left\{f_{n}\right\}$ is normal. Thus we have proved that $X$ is taut modulo $\operatorname{Sp}(X)$.

We remark that Theorem 1.4 conversely implies Theorem 2.1. Indeed suppose contrary, that there exists a sequence $\left\{f_{n}\right\}$ in $\operatorname{Hol}(\mathbb{D}, X)$ such that $f_{n}(\mathbb{D}(s)) \not \subset U$ for all $n$, but $\left|f_{n}^{\prime}(0)\right|_{\omega_{A}} \rightarrow \infty$ as $n \rightarrow \infty$. Then Theorem 1.4 implies that the sequence $\left\{f_{n}\right\}$ is normal. Hence after taking a subsequence, the sequence $\left\{f_{n}\right\}$ converges locally uniformly to a holomorphic map $f: \mathbb{D} \rightarrow X$. Hence $\left|f_{n}^{\prime}(0)\right|_{\omega_{A}} \rightarrow\left|f^{\prime}(0)\right|_{\omega_{A}}$ as $n \rightarrow \infty$. This is a contradiction.

The following is a counter example for the converse of the theorem of Kiernan and Kobayashi.

EXAMPLE 2.2. Let $X$ be a closed subvariety of an abelian variety such that $\operatorname{Sp}(X)=\emptyset$. Assume that $d=\operatorname{dim} X \geq 2$. Let $p: \tilde{X} \rightarrow X$ be the blow-up of $X$ along a smooth point $x \in X$. Set $\Delta=p^{-1}(x)$. Then, according to the theorem of Green (or Theorem 1.2), it is easy to see that $\tilde{X}$ is Kobayashi hyperbolic modulo $\Delta$. However, $\tilde{X}$ is not taut modulo $\Delta$. Indeed, let $\left(y_{1}, \ldots, y_{d}\right) \in \mathbb{D}^{d}$ be a local coordinate around $x \in X$ such that the origin of $\mathbb{D}^{d}$ corresponds to $x$. Let $f_{n}: \mathbb{D} \rightarrow X$ be defined by $f_{n}(z)=(z, 1 / 2 n, 0, \ldots, 0)$. Then the sequence $\left\{f_{n}\right\}$ converges locally uniformly to $f(z)=(z, 0, \ldots, 0)$. However, if we denote by $\tilde{f}_{n}: \mathbb{D} \rightarrow \tilde{X}$ the lift of $f_{n}$, we have $\left|\tilde{f}_{n}^{\prime}(0)\right| \rightarrow \infty$ as $n \rightarrow \infty$. In particular, any subsequence of the sequence $\left\{\tilde{f}_{n}\right\}$ does not converge locally uniformly to the lift $\tilde{f} \in \operatorname{Hol}(\mathbb{D}, \tilde{X})$ of $f$. This shows that $\tilde{X}$ is not taut modulo $\Delta$.

This example shows that the procedure of blowing-up may cause a delicate problem when we consider the norm of the derivatives of holomorphic maps. In the proof of Theorem 2.1, we need to take several blowing-ups. These actually cause main technical issues in the proof.

Now we introduce the main proposition (cf. Proposition 2.3) in the proof of Theorem 2.1. Although the statement of this proposition is rather complicated, it states a Nevanlinna theoretic version of Theorem 2.1 in relative setting for a family $X \subset A \times S$ of closed subvarieties of an abelian variety $A$ over a smooth projective variety $S$.

For a positive dimensional abelian variety $A$, we denote by $\Sigma(A)$ the set of all proper abelian subvarieties of $A$ including the trivial one $\{0\} \subset A$. Hence we remark that $A \notin \Sigma(A)$ and $\{0\} \in \Sigma(A)$.

Conventions. Let $V_{1}$ and $V_{2}$ be algebraic varieties and let $f \in \operatorname{Hol}\left(\mathbb{D}, V_{1} \times V_{2}\right)$. Then we denote by $f_{V_{1}} \in \operatorname{Hol}\left(\mathbb{D}, V_{1}\right)$ and $f_{V_{2}} \in \operatorname{Hol}\left(\mathbb{D}, V_{2}\right)$ the compositions with $f$ and the first projection $p_{1}: V_{1} \times V_{2} \rightarrow V_{1}$ and the second projection $p_{2}: V_{1} \times V_{2} \rightarrow V_{2}$, 
respectively. If $\omega_{V_{1}}$ and $\omega_{V_{2}}$ are $(1,1)$-forms on $V_{1}$ and $V_{2}$, respectively, then we set $\omega_{V_{1} \times V_{2}}=p_{1}^{*} \omega_{V_{1}}+p_{2}^{*} \omega_{V_{2}}$.

Proposition 2.3. Let $A$ be a positive dimensional abelian variety and let $S$ be a smooth projective variety. Let $X \subset A \times S$ be a closed subscheme such that for every $y \in S$, the fiber $X_{y}$ over $y$ satisfies $X_{y} \varsubsetneqq A$. Then there exists a non-empty finite subset $\Lambda \subset \Sigma(A)$ with the following property: Let $\omega_{A}$ be a positive invariant $(1,1)$-form on $A$, and let $\omega_{S}$ be a smooth, positive $(1,1)$-form on $S$. For each $B \in \Sigma(A)$, let $\omega_{A / B}$ be a positive invariant $(1,1)$-form on $A / B$ and $\varpi_{B}: A \rightarrow A / B$ be the quotient map. Let $0<s<1, \varepsilon>0$ and $\delta>0$. Then there exist positive constants $c_{1}, c_{2}, c_{3}$ such that, for every $f \in \operatorname{Hol}(\mathbb{D}, X)$, the estimate

$$
\begin{aligned}
\min _{B \in \Lambda}\left\{\int_{s}^{r} d t \int_{\mathbb{D}(t)}\left(\varpi_{B} \circ f_{A}\right)^{*} \omega_{A / B}\right\} \leq & \varepsilon \int_{s}^{r} d t \int_{\mathbb{D}(t)} f_{A}^{*} \omega_{A}+c_{1} \int_{s}^{r} d t \int_{\mathbb{D}(t)} f_{S}^{*} \omega_{S} \\
& +c_{2} \max \left\{0, \int_{0}^{2 \pi} \log \frac{1}{\left|f^{\prime}\left(s e^{i \theta}\right)\right|_{\omega_{A \times S}}} \frac{d \theta}{2 \pi}\right\}+c_{3}
\end{aligned}
$$

holds for all $r \in(s, 1)$ outside some exceptional set $E \subset(s, 1)$ with the linear measure $|E|<\delta$.

$\operatorname{Here} \operatorname{Hol}(\mathbb{D}, X)$ is the set of all holomorphic mappings $\mathbb{D} \rightarrow X$, where $X$ is considered as a possibly non-reduced and reducible complex space. Hence $\operatorname{Hol}(\mathbb{D}, X)=\{f \in$ $\operatorname{Hol}(\mathbb{D}, A \times S) ; f(\mathbb{D}) \subset \operatorname{supp} X\}$. In the statement of Proposition 2.3, we only need to consider the support of $X$. However we consider the scheme structure of $X$ for the sake of convenience in the proof.

The outline of the proof of Proposition 2.3 is as follows. The proof is done by transfinite induction with respect to the maximum Hilbert polynomial attached to the family $X \rightarrow S$. In Section 3, we prove an algebro-geometric lemma (cf. Lemma 3.1), which states that this maximum Hilbert polynomial reduces by blowing-up the base space $S$ by a closed subscheme $\mathcal{T} \subset S$. Here the support of $\mathcal{T}$ is the locus $T \subset S$ over which the Hilbert polynomials are maximum. At first glance, this process works well to complete the transfinite induction for the proof of Proposition 2.3, but this is not the case; If the image $f_{S}(\mathbb{D})$ is very close to $T$, we can not get the desired estimate for $f: \mathbb{D} \rightarrow A \times S$ using the induction hypothesis for the lift $\mathbb{D} \rightarrow A \times \mathrm{Bl}_{\mathcal{T}} S$ of $f$ (cf. Example 2.2). Here $\mathrm{Bl}_{\mathcal{T}} S$ is the blow-up of $S$ along $\mathcal{T}$. To handle this problem, we consider Demailly jet space $A \times S_{[\nu]}$ of $A \times S$ with sufficiently high order $\nu$, which has a property described in Lemma 4.2 (cf. Section 4). By the tautological inequality (cf. Section 5), we may reduce the estimate of Proposition 2.3 for holomorphic maps $f: \mathbb{D} \rightarrow A \times S$ to that of their jet lifts $f_{[\nu]}: \mathbb{D} \rightarrow A \times S_{[\nu]}$. We consider the jet space $X_{\nu} \subset A \times S_{[\nu]}$ of $X$ as a family of closed subschemes of $A$ over $S_{[\nu]}$. By Lemma 3.1 mentioned above, we may reduce the maximum Hilbert polynomial after blowing-up $S_{[\nu]}$ by $T_{[\nu]} \subset S_{[\nu]}$ with a prescribed closed subscheme structure $\mathcal{T}$. Here $T_{[\nu]} \subset S_{[\nu]}$ is the locus over which the Hilbert polynomials are maximum. Now we need to consider the two cases according to whether or not the image $\left(f_{[\nu]}\right)_{S_{[\nu]}}(\mathbb{D}(r))$ is almost contained in a neighborhood of $T_{[\nu]}$. Here the terminology almost is introduced in Section 6 . If this is the case, then without using the 
induction hypothesis, the desired estimate follows from the geometric conclusion about the Demailly jet spaces (cf. Lemma 4.4) and an application of Vitali covering theorem (cf. Section 7). Next if $\left(f_{[\nu]}\right)_{S_{[\nu]}}(\mathbb{D}(r))$ is not almost contained in the neighborhood of $T_{[\nu]}$, we apply the induction hypothesis. In this case, the main issue is to get the estimate for $f_{[\nu]}: \mathbb{D} \rightarrow A \times S_{[\nu]}$ from the estimate obtained by the induction hypothesis for the lift $\mathbb{D} \rightarrow A \times \mathrm{Bl}_{\mathcal{T}} S_{[\nu]}$ of $f_{[\nu]}$. This is done by applying the Bloch-Cartan estimate (cf. Lemma 6.1).

\section{Hilbert polynomial and blowing-up.}

Let $S$ be a variety (cf. Remark 1.6). Let $X \subset \mathbb{P}^{n} \times S$ be a closed subscheme and let $p: X \rightarrow S$ be the composite of the closed immersion $X \hookrightarrow \mathbb{P}^{n} \times S$ and the second projection $\mathbb{P}^{n} \times S \rightarrow S$. Let $\mathcal{L}$ be a relatively very ample invertible sheaf on $X$ which is obtained by the pull-back of $\mathcal{O}_{\mathbb{P} n}(1)$ by the composite of $X \hookrightarrow \mathbb{P}^{n} \times S$ and the first projection $\mathbb{P}^{n} \times S \rightarrow \mathbb{P}^{n}$. For $s \in S$, let $X_{s} \subset \mathbb{P}^{n}$ be the fiber over $s$, and $\mathcal{L}_{s}$ be the restriction of $\mathcal{L}$ on $X_{s}$. We denote by $P_{X_{s}}$ the Hilbert polynomial of $X_{s}$ with respect to $\mathcal{L}_{s}$. Hence for $m \gg 1$, we have

$$
P_{X_{s}}(m)=\operatorname{dim} H^{0}\left(X_{s}, \mathcal{L}_{s}^{\otimes m}\right) .
$$

Then the set $\left\{P_{X_{s}}\right\}_{s \in S}$ is finite (cf. [31, p.201, Step 2]). Since $S \neq \emptyset$, the set $\left\{P_{X_{s}}\right\}_{s \in S}$ is non-empty.

Let $\mathcal{P}$ be the set of all numerical polynomials which appear as Hilbert polynomials of closed subschemes of the projective space $\mathbb{P}^{n}$. Then $\mathcal{P}$ is an ordered set by $P_{1} \leq P_{2}$ if and only if $P_{1}(m) \leq P_{2}(m)$ for all large integers $m$. This order is a total order. We may take the maximum element $P_{\max }$ from the non-empty, finite set $\left\{P_{X_{s}}\right\}_{s \in S} \subset \mathcal{P}$ with respect to this order. We set

$$
T= \begin{cases}\left\{s \in S ; P_{X_{s}}=P_{\max }\right\} & \text { if } X \neq \emptyset, \\ \emptyset & \text { if } X=\emptyset .\end{cases}
$$

We remark that $T \subset p(X)$.

Lemma 3.1. (1) $T$ is a Zariski closed subset of $S$.

(2) Assume $T \neq S$. Then there exists a closed subscheme $\mathcal{T} \subset S$ such that $\operatorname{supp} \mathcal{T}=$ $T$ with the following property: Let $\alpha: \mathrm{Bl}_{\mathcal{T}} S \rightarrow S$ be the blow-up along $\mathcal{T}$, let $\hat{X} \subset$ $\mathbb{P}^{n} \times \mathrm{Bl}_{\mathcal{T}} S$ be the scheme-theoretic closure of $p^{-1}(S-T)$ in $X \times{ }_{S} \mathrm{Bl}_{\mathcal{T}} S \subset \mathbb{P}^{n} \times \mathrm{Bl}_{\mathcal{T}} S$. Let $\hat{p}: \hat{X} \rightarrow \mathrm{Bl}_{\mathcal{T}} S$ be the composite of the closed immersion $\hat{X} \hookrightarrow \mathbb{P}^{n} \times \mathrm{Bl}_{\mathcal{T}} S$ and the second projection $\mathbb{P}^{n} \times \mathrm{Bl}_{\mathcal{T}} S \rightarrow \mathrm{Bl}_{\mathcal{T}} S$. Then, for every $t \in \mathrm{Bl}_{\mathcal{T}} S$ such that $\alpha(t) \in T$, the closed immersion $\hat{X}_{t} \hookrightarrow X_{\alpha(t)}$ is not an isomorphism, where $\hat{X}_{t}$ is the fiber of $\hat{p}: \hat{X} \rightarrow \mathrm{Bl}_{\mathcal{T}} S$ over $t \in \mathrm{Bl}_{\mathcal{T}} S$.

(3) Assume $T \neq S$ and $X \neq \emptyset$. Let $\hat{p}: \hat{X} \rightarrow \mathrm{Bl}_{\mathcal{T}} S$ be the object described in the previous assertion. Set $\hat{P}=\max _{s \in B_{\mathcal{T}} S}\left\{P_{\hat{X}_{s}}\right\}$ and $P=\max _{s \in S}\left\{P_{X_{s}}\right\}$. Then $\hat{P}<P$.

REMARK 3.2. Assume that $S$ is smooth. Then in the assertion (2), we may take $\mathcal{T} \subset S$ so that $\tilde{S}=\mathrm{Bl}_{\mathcal{T}} S$ is also smooth. Indeed, if $\tilde{S}$ is not smooth, we replace $\tilde{S}$ by a 
smooth model $\tilde{\tilde{S}} \rightarrow \tilde{S}$ which is isomorphic outside the support of $\alpha^{*} \mathcal{T}$.

Proof. When $X=\emptyset$, our assertions (1) and (2) are trivial. In the following, we assume $X \neq \emptyset$.

We first prove (1). By [31, p.201, Step 3], there exists $N_{1}>0$ such that for every $s \in S$ and every $m \geq N_{1}$, the natural morphism

$$
\left(p_{*} \mathcal{L}^{\otimes m}\right) \otimes \mathbb{C}(s) \rightarrow \Gamma\left(X_{s}, \mathcal{L}_{s}^{\otimes m}\right)
$$

is an isomorphism, and

$$
H^{j}\left(X_{s}, \mathcal{L}_{s}^{\otimes m}\right)=\{0\}
$$

for all $j>0$. Hence $P_{X_{s}}(m)=\operatorname{dim}\left\{\left(p_{*} \mathcal{L}^{\otimes m}\right) \otimes \mathbb{C}(s)\right\}$ for every $s \in S$ and every $m \geq N_{1}$. Replacing $N_{1}$ by a lager integer if necessary, we may assume, moreover, that $P_{X_{s}}(m)<$ $P_{\max }(m)$ for every $s \in S-T$ and every $m \geq N_{1}$. The function $s \mapsto \operatorname{dim}\left\{\left(p_{*} \mathcal{L}^{\otimes N_{1}}\right) \otimes \mathbb{C}(s)\right\}$ is upper semicontinuous and the locus where the value of this function is greater than or equal to $P_{\max }\left(N_{1}\right)$ is exactly equal to $T$. Hence $T$ is Zariski closed. This shows (1).

Next we prove (2). We apply the stratification defined by $p_{*} \mathcal{L}^{\otimes m}([\mathbf{3 1}$, Theorem 4.2.7]). For $m \geq N_{1}$, we obtain a closed subscheme $\mathcal{T}_{m} \subset S$ such that $\operatorname{supp} \mathcal{T}_{m}=T$ with the following property: If $q: V \rightarrow S$ is a scheme morphism, the sheaf $q^{*}\left(p_{*} \mathcal{L}^{\otimes m}\right)$ is locally free of rank $P_{\max }(m)$ if and only if $q$ factors through $\mathcal{T}_{m}$. We set $\mathcal{T}=\bigcap_{m \geq N_{1}} \mathcal{T}_{m}$. By the Noetherian property, $\mathcal{T}$ is well-defined closed subscheme of $S$ with $\operatorname{supp} \underset{\mathcal{T}}{=} T$.

We shall show that $\mathcal{T}$ satisfies the property of (2). Let $\alpha: \tilde{S} \rightarrow S$ be the blow-up along $\mathcal{T}$. Set $\tilde{X}=X \times_{S} \tilde{S}$ and let $\tilde{p}: \tilde{X} \rightarrow \tilde{S}$ be the projection. Let $\tilde{\mathcal{L}}$ be the pull-back of $\mathcal{L}$ by $\tilde{X} \rightarrow X$. Then $\tilde{\mathcal{L}}$ is a relatively very ample invertible sheaf. Let $\alpha^{*} \mathcal{T}$ be the pull-back of $\mathcal{T}$ by $\alpha$. Then $\alpha^{*} \mathcal{T} \subset \tilde{S}$ is a Cartier divisor.

We construct a closed subscheme $\tilde{\mathcal{T}} \subset \tilde{S}$ in a similar manner as the construction of $\mathcal{T} \subset S$ above. We take $N_{2} \geq N_{1}$ such that the natural morphism

$$
\left(\tilde{p}_{*} \tilde{\mathcal{L}}^{\otimes m}\right) \otimes \mathbb{C}(s) \rightarrow \Gamma\left(\tilde{X}_{s}, \tilde{\mathcal{L}}_{s}^{\otimes m}\right)
$$

is an isomorphism for every $m \geq N_{2}$ and every $s \in \tilde{S}$. Replacing $N_{2}$ by a lager integer if necessary, we may assume, moreover, that the natural map

$$
\alpha^{*}\left(p_{*} \mathcal{L}^{\otimes m}\right) \rightarrow \tilde{p}_{*} \tilde{\mathcal{L}}^{\otimes m}
$$

is an isomorphism for every $m \geq N_{2}$ (cf. [31, Proposition 4.2.4]). For $m \geq N_{2}$, we obtain a closed subscheme $\tilde{\mathcal{T}}_{m} \subset \tilde{S}$ such that supp $\tilde{\mathcal{T}}_{m}=\operatorname{supp} \alpha^{*} \mathcal{T}$ with the following property: If $q: V \rightarrow \tilde{S}$ is a scheme morphism, the sheaf $q^{*}\left(\tilde{p}_{*} \tilde{\mathcal{L}}^{\otimes m}\right)$ is locally free of rank $P_{\max }(m)$ if and only if $q$ factors through $\tilde{\mathcal{T}}_{m}$. We set $\tilde{\mathcal{T}}=\bigcap_{m \geq N_{2}} \tilde{\mathcal{T}}_{m}$. Then $\tilde{\mathcal{T}}$ is a closed subscheme such that $\operatorname{supp} \tilde{\mathcal{T}}=\operatorname{supp} \alpha^{*} \mathcal{T}$.

We shall show $\tilde{\mathcal{T}}=\alpha^{*} \mathcal{T}$ to conclude that $\tilde{\mathcal{T}} \subset \tilde{S}$ is a Cartier divisor. Let $q: \alpha^{*} \mathcal{T} \rightarrow \tilde{S}$ be the closed immersion. Then $\alpha \circ q: \alpha^{*} \mathcal{T} \rightarrow S$ factors through $\mathcal{T}_{m}$ for every $m \geq N_{1}$. Hence $(\alpha \circ q)^{*}\left(p_{*} \mathcal{L}^{\otimes m}\right)$ is locally free of rank $P_{\max }(m)$ for every $m \geq N_{1}$. For $m \geq N_{2}$, (3.4) yields $(\alpha \circ q)^{*}\left(p_{*} \mathcal{L}^{\otimes m}\right)=q^{*}\left(\tilde{p}_{*} \tilde{\mathcal{L}}^{\otimes m}\right)$. Hence $q^{*}\left(\tilde{p}_{*} \tilde{\mathcal{L}}^{\otimes m}\right)$ is locally free of rank 
$P_{\max }(m)$ for every $m \geq N_{2}$. Hence $q$ factors through $\tilde{\mathcal{T}}_{m} \subset \tilde{S}$ for every $m \geq N_{2}$. This shows $\alpha^{*} \mathcal{T} \subset \tilde{\mathcal{T}}$.

Next we shall show $\tilde{\mathcal{T}} \subset \alpha^{*} \mathcal{T}$. Let $\psi: \tilde{\mathcal{T}} \rightarrow \tilde{S}$ be the closed immersion. Set $X_{\tilde{\mathcal{T}}}=\tilde{X} \times_{\tilde{S}} \tilde{\mathcal{T}}$ and let $p_{\tilde{\mathcal{T}}}: X_{\tilde{\mathcal{T}}} \rightarrow \tilde{\mathcal{T}}$ be the projection. Let $\mathcal{L}_{\tilde{\mathcal{T}}}$ be the pull back of $\tilde{\mathcal{L}}$ on $X_{\tilde{\mathcal{T}}}$.

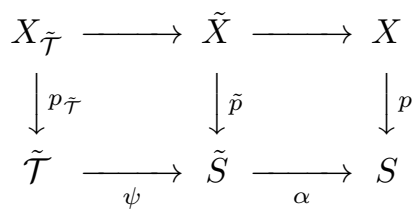

There exists a positive integer $N_{3} \geq N_{2}$ such that the natural map

$$
\psi^{*}\left(\tilde{p}_{*} \tilde{\mathcal{L}}^{\otimes m}\right) \rightarrow\left(p_{\tilde{\mathcal{T}}}\right)_{*} \mathcal{L}_{\tilde{\mathcal{T}}}^{\otimes m}
$$

is an isomorphism for every $m \geq N_{3}$ (cf. [31, Proposition 4.2.4]). Hence $\left(p_{\tilde{\mathcal{T}}}\right)_{*} \mathcal{L}_{\tilde{\mathcal{T}}}^{\otimes m}$ is locally free for every $m \geq N_{3}$. Hence by [31, Proposition 4.2.1], $p_{\tilde{\mathcal{T}}}: X_{\tilde{\mathcal{T}}} \rightarrow \tilde{\mathcal{T}}$ is flat. Now we look at the natural map

$$
(\alpha \circ \psi)^{*} p_{*} \mathcal{L}^{\otimes m} \rightarrow\left(p_{\tilde{\mathcal{T}}}\right)_{*} \mathcal{L}_{\tilde{\mathcal{T}}}^{\otimes m}
$$

Then in view of (3.2), the natural map

$$
\left(p_{\tilde{\mathcal{T}}}\right)_{*} \mathcal{L}_{\tilde{\mathcal{T}}}^{\otimes m} \otimes \mathbb{C}(s) \rightarrow \Gamma\left(\left(X_{\tilde{\mathcal{T}}}\right)_{s},\left(\mathcal{L}_{\tilde{\mathcal{T}}}\right)_{s}^{\otimes m}\right)
$$

is surjective for every $m \geq N_{1}$ and $s \in \tilde{\mathcal{T}}$. We apply the theorem of cohomology and base change to conclude that (3.6) is an isomorphism for every $m \geq N_{1}$ and $s \in \tilde{\mathcal{T}}$ (cf. $[\mathbf{1 2}$, Chapter III, Theorem $12.11(\mathrm{a})])$, and $\left(p_{\tilde{\mathcal{T}}}\right)_{*} \mathcal{L}_{\tilde{\mathcal{T}}}^{\otimes m}$ is locally free of rank $P_{\max }(m)$ for every $m \geq N_{1}$ (cf. [12, Chapter III, Theorem 12.11 (b)]). By the isomorphisms (3.2) and (3.6), the natural map

$$
(\alpha \circ \psi)^{*} p_{*} \mathcal{L}^{\otimes m} \otimes \mathbb{C}(s) \rightarrow\left(p_{\tilde{\mathcal{T}}}\right)_{*} \mathcal{L}_{\tilde{\mathcal{T}}}^{\otimes m} \otimes \mathbb{C}(s)
$$

induced by $(3.5)$ is an isomorphism for every $m \geq N_{1}$ and $s \in \tilde{\mathcal{T}}$. Since $\left(p_{\tilde{\mathcal{T}}}\right)_{*} \mathcal{L}_{\tilde{\mathcal{T}}}^{\otimes m}$ is locally free, Nakayama's lemma yields that (3.5) is an isomorphism for every $m \geq N_{1}$. Hence $(\alpha \circ \psi)^{*} p_{*} \mathcal{L}^{\otimes m}$ is locally free of rank $P_{\max }(m)$ for every $m \geq N_{1}$. This shows that $\alpha \circ \psi: \tilde{\mathcal{T}} \rightarrow S$ factors through $\mathcal{T}$. Hence $\tilde{\mathcal{T}} \subset \alpha^{*} \mathcal{T}$. We have proved $\tilde{\mathcal{T}}=\alpha^{*} \mathcal{T}$.

We take $t \in \operatorname{supp} \tilde{\mathcal{T}}$. Then for $m \geq N_{2}$, we have an exact sequence on a Zariski open neighborhood $U_{m}$ of $t$,

$$
\mathcal{O}_{\tilde{S}}^{\oplus d_{m}} \stackrel{G_{m}}{\rightarrow} \mathcal{O}_{\tilde{S}}^{\oplus e_{m}} \rightarrow \tilde{p}_{*} \tilde{\mathcal{L}}^{\otimes m} \rightarrow 0
$$

such that the induced

$$
\mathcal{O}_{\tilde{S}}^{\oplus e_{m}} \otimes \mathbb{C}(t) \rightarrow \tilde{p}_{*} \tilde{\mathcal{L}}^{\otimes m} \otimes \mathbb{C}(t)
$$


is an isomorphism. We take a positive integer $l$ such that $\tilde{\mathcal{T}}=\tilde{\mathcal{T}}_{N_{2}} \cap \cdots \cap \tilde{\mathcal{T}}_{N_{2}+l}$. We take an affine open neighborhood $U$ of $t$ such that $U \subset U_{m}$ for $m=N_{2}, \ldots, N_{2}+l$. We may assume that $\tilde{\mathcal{T}} \cap U$ is defined by the ideal $\sigma \cdot \mathcal{O}_{\tilde{S}}(U) \subset \mathcal{O}_{\tilde{S}}(U)$ for some $\sigma \in \mathcal{O}_{\tilde{S}}(U)$. Since $\tilde{S}$ is a variety (cf. [12, Chapter II, Proposition 7.16]) and $\operatorname{supp} \tilde{\mathcal{T}} \neq \tilde{S}$, we have $\sigma \neq 0$ as elements of $\mathcal{O}_{\tilde{S}}(U)$. Let $G_{m}$ be expressed by the matrix $\left(g_{i j}^{(m)}\right)$. We denote by $I_{m} \subset \mathcal{O}_{\tilde{S}}(U)$ the ideal generated by all $g_{i j}^{(m)}$, where $1 \leq i \leq e_{m}$ and $1 \leq j \leq d_{m}$. Then $I_{m}$ is the ideal associated to the closed subscheme $\tilde{\mathcal{T}}_{m} \cap U \subset U$. Hence we have $I_{N_{2}}+\cdots+I_{N_{2}+l}=\sigma \cdot \mathcal{O}_{\tilde{S}}(U)$. We may write $g_{i j}^{(m)}=\sigma h_{i j}^{(m)}$ by $h_{i j}^{(m)} \in \mathcal{O}_{\tilde{S}}(U)$. Since $\mathcal{O}_{\tilde{S}}(U)$ is an integral domain, there exists $\mu_{i j}^{(m)} \in \mathcal{O}_{\tilde{S}}(U)$ such that

$$
\sum \mu_{i j}^{(m)} h_{i j}^{(m)}=1
$$

Hence there exists $h_{i^{\prime} j^{\prime}}^{\left(m^{\prime}\right)}$ such that $h_{i^{\prime} j^{\prime}}^{\left(m^{\prime}\right)}(t) \neq 0$. Let $\tau \in \Gamma\left(U, \tilde{p}_{*} \tilde{\mathcal{L}}^{\otimes m^{\prime}}\right)$ be the image of $\left(h_{1 j^{\prime}}^{\left(m^{\prime}\right)}, \ldots, h_{e_{m}, j^{\prime}}^{\left(m^{\prime}\right)}\right)$ under (3.7). Then $\sigma \tau=0$, but $\left.\tau\right|_{t} \neq 0$, where we consider $\left.\tau\right|_{t} \in$ $\Gamma\left(\tilde{X}_{t}, \tilde{\mathcal{L}}_{t}^{\otimes m^{\prime}}\right)$ under the isomorphisms (3.3) and (3.8). Let

$$
V(\tau) \hookrightarrow \tilde{p}^{-1}(U)
$$

be a closed subscheme defined by $\tau=0$, where $\tau \in \Gamma\left(\tilde{p}^{-1}(U), \tilde{\mathcal{L}}^{\otimes m^{\prime}}\right)$. Since $\sigma$ is a unit on $U-\operatorname{supp} \tilde{\mathcal{T}}$, the immersion $(3.9)$ is an isomorphism over $U-\operatorname{supp} \tilde{\mathcal{T}}$. Hence we have $\hat{X} \cap \tilde{p}^{-1}(U) \subset V(\tau)$. Here we remark that $\hat{X} \cap \tilde{p}^{-1}(U)$ is the scheme theoretic closure of $\tilde{p}^{-1}(U-\operatorname{supp} \tilde{\mathcal{T}})$ in $\tilde{p}^{-1}(U)$, because the scheme theoretic closure of a Noetherian scheme commutes with restriction to open subset. On the other hand, by $\left.\tau\right|_{t} \neq 0$, we have $V(\tau)_{t} \varsubsetneqq \tilde{X}_{t}$. Hence we have

$$
\hat{X}_{t} \subset V(\tau)_{t} \varsubsetneqq \tilde{X}_{t}=X_{\alpha(t)} .
$$

This completes the proof of Lemma 3.1 (2). (Compare with the proof of $[\mathbf{1 4}$, Theorem 1.14].)

Before going to prove (3), we prepare another lemma.

LEMma 3.3. Let $Y \subset \mathbb{P}^{N}$ be a closed subscheme and let $Z \subset Y$ be a closed subscheme. Then $P_{Z} \leq P_{Y}$, where $P_{Y}$ and $P_{Z}$ are the Hilbert polynomials of $Y$ and $Z$, respectively. If, moreover, the closed immersion $Z \hookrightarrow Y$ is not an isomorphism, then $P_{Z}<P_{Y}$.

Proof of Lemma 3.3. Let $\mathcal{I} \subset \mathcal{O}_{Y}$ be the ideal sheaf of $Z \subset Y$. Then we have the following short exact sequence

$$
0 \longrightarrow \mathcal{I} \longrightarrow \mathcal{O}_{Y} \longrightarrow \mathcal{O}_{Z} \longrightarrow 0 \text {. }
$$

By $H^{1}(Y, \mathcal{I}(m))=0$ for $m \gg 1$, this sequence yields

$$
0 \longrightarrow \Gamma(Y, \mathcal{I}(m)) \longrightarrow \Gamma\left(Y, \mathcal{O}_{Y}(m)\right) \longrightarrow \Gamma\left(Y, \mathcal{O}_{Z}(m)\right) \longrightarrow 0
$$

for $m \gg 1$. By $P_{Y}(m)=\operatorname{dim} \Gamma\left(Y, \mathcal{O}_{Y}(m)\right)$ and $P_{Z}(m)=\operatorname{dim} \Gamma\left(Y, \mathcal{O}_{Z}(m)\right)$ for $m \gg 1$, 
we get

$$
P_{Y}(m)=P_{Z}(m)+\operatorname{dim} \Gamma(Y, \mathcal{I}(m))
$$

for $m \gg 1$. Thus $P_{Z} \leq P_{Y}$.

Now assume moreover $Z \neq Y$. Then $\mathcal{I} \neq 0$. Since $\mathcal{I}(m)$ is generated by its global sections for $m \gg 1$, we get $\Gamma(Y, \mathcal{I}(m)) \neq\{0\}$ for $m \gg 1$. Hence $P_{Z}<P_{Y}$.

Now we return to the proof of Lemma 3.1 (3). We take $t \in \mathrm{Bl}_{\mathcal{T}} S$ such that $P_{\hat{X}_{t}}=\hat{P}$. The proof is divided in the two cases whether $\alpha(t) \in T$ or not. If $\alpha(t) \in T$, then by Lemma 3.1 (2), we have $\hat{X}_{t} \varsubsetneqq X_{\alpha(t)}$. Thus by Lemma 3.3 , we have $\hat{P}=P_{\hat{X}_{t}}<P_{X_{\alpha(t)}}=P$. If $\alpha(t) \notin T$, then the assumption $X \neq \emptyset$ yields $P_{X_{\alpha(t)}}<P$. Hence $\hat{P}=P_{\hat{X}_{t}} \leq P_{X_{\alpha(t)}}<P$. In both cases, $\hat{P}<P$. This completes the proof of Lemma 3.1 (3).

\section{Demailly jet spaces.}

We introduce Demailly jet spaces (cf. [6]). Let $M$ be a positive dimensional smooth algebraic variety. Let $V \subset T M$ be an algebraic vector subbundle, whose bundle rank is positive. Set $\tilde{M}=P(V)$. Let $\pi: \tilde{M} \rightarrow M$ be the projection. We define a vector subbundle $\tilde{V} \subset T \tilde{M}$ as follows: For every point $(x,[v]) \in \tilde{M}$ associated with a vector $v \in V_{x} \backslash\{0\}$, we set

$$
\tilde{V}_{(x,[v])}=\left\{\xi \in T_{(x,[v])} \tilde{M} ; \pi_{*}(\xi) \in \mathbb{C} v\right\}
$$

Let $f: \mathbb{D} \rightarrow M$ be a non-constant holomorphic map. We say that $f$ is tangent to $V$ if $f^{\prime}(z) \in V_{f(z)}$ for all $z \in \mathbb{D}$. If $f$ is tangent to $V$, we may define $f_{[1]}: \mathbb{D} \rightarrow \tilde{M}$ by $f_{[1]}(z)=\left(f(z),\left[f^{\prime}(z)\right]\right)$. Then $f_{[1]}$ is tangent to $\tilde{V}$.

Let $Z \subset M$ be a closed subscheme. We define a closed subscheme $\tilde{Z} \subset \tilde{M}$ as follows. Let $W \subset M$ be an affine open set where $Z \cap W$ is defined by $\phi_{1}, \ldots, \phi_{l} \subset \Gamma\left(W, \mathcal{O}_{W}\right)$. Then we define the closed subscheme $\widetilde{Z \cap W} \subset \pi^{-1}(W)$ by $\phi_{1}, \ldots, \phi_{l},\left.d \phi_{1}\right|_{V}, \ldots,\left.d \phi_{l}\right|_{V}$. Then this definition of $\widetilde{Z \cap W}$ does not depend on the choice of generators $\phi_{1}, \ldots, \phi_{l}$, so well defined over $W$. In general, we cover $M$ by open affines $\left\{W_{i}\right\}$ and make closed subschemes $\widetilde{Z \cap W_{i}} \subset \pi^{-1}\left(W_{i}\right)$. Then we glue these subschemes and define the subscheme $\tilde{Z} \subset \tilde{M}$.

We inductively define the Demailly jet space $M_{k}$ together with a vector subbundle $V_{k} \subset T M_{k}$ by

$$
\left(M_{0}, V_{0}\right)=(M, T M), \quad\left(M_{k}, V_{k}\right)=\left(\widetilde{M_{k-1}}, \widetilde{V_{k-1}}\right) .
$$

For a non-constant holomorphic map $f: \mathbb{D} \rightarrow M$, we define $f_{[k]}: \mathbb{D} \rightarrow M_{k}$ inductively by $f_{[0]}=f$ and $f_{[k]}=\left(f_{[k-1]}\right)_{[1]}$. For a closed subscheme $Z \subset M$, we define a closed subscheme $Z_{k} \subset M_{k}$ inductively by $Z_{0}=Z$ and $Z_{k}=\widetilde{Z_{k-1}}$. Then for each non-constant $f \in \operatorname{Hol}(\mathbb{D}, Z)$, we have $f_{[k]} \in \operatorname{Hol}\left(\mathbb{D}, Z_{k}\right)$ for all $k \geq 0$.

Now let $A$ be a non-trivial abelian variety, let $S$ be a smooth projective variety, and let $M=A \times S$. We denote by $\operatorname{Lie}(A)$ the tangent space to $A$ at the identity point $0 \in A$. Let $T S \times \operatorname{Lie}(A) \rightarrow S$ be the composite of the first projection $T S \times \operatorname{Lie}(A) \rightarrow T S$ and 
the natural projection $T S \rightarrow S$. We then consider $T S \times \operatorname{Lie}(A) \rightarrow S$ as a vector bundle of $\operatorname{rank} \operatorname{dim} M$ over $S$. Then $T M=q^{*}(T S \times \operatorname{Lie}(A))$, where $q: M \rightarrow S$ is the second projection.

Lemma 4.1. For each $k \geq 0$, there exist a smooth projective variety $S_{[k]}$ and a vector subbundle $V_{k}^{\dagger} \subset T S_{[k]} \times \operatorname{Lie}(A)$ such that $M_{k}=A \times S_{[k]}$ and $V_{k}=q_{k}^{*} V_{k}^{\dagger} \subset T M_{k}$, where $q_{k}: M_{k} \rightarrow S_{[k]}$ is the second projection.

Proof. We prove by induction on $k \geq 0$. Our assertion is valid for $k=0$, where $S_{[0]}=S$ and $V_{0}^{\dagger}=T S \times \operatorname{Lie}(A)$. For the induction step, suppose our assertion is valid for $k-1$. We set $S_{[k]}=P\left(V_{k-1}^{\dagger}\right)$. Then $S_{[k]}$ is a smooth projective variety. We have

$$
M_{k}=P\left(q_{k-1}^{*}\left(V_{k-1}^{\dagger}\right)\right)=M_{k-1} \times_{S_{[k-1]}} S_{[k]}=A \times S_{[k]} .
$$

Next let $\tau: S_{[k]} \rightarrow S_{[k-1]}$ be the projection. We have a vector bundle map $\left(\tau_{*}, \operatorname{id} \operatorname{die}(A)_{\text {(ie }}\right)$ : $T S_{[k]} \times \operatorname{Lie}(A) \rightarrow T S_{[k-1]} \times \operatorname{Lie}(A)$. We define $V_{k}^{\dagger} \subset T S_{[k]} \times \operatorname{Lie}(A)$ as follows. For each $(x,[v]) \in S_{[k]}$, where $x \in S_{[k-1]}$ and $v \in V_{k-1}^{\dagger} \backslash\{0\}$, we set

$$
\left(V_{k}^{\dagger}\right)_{(x,[v])}=\left\{\xi \in T_{(x,[v])} S_{[k]} \times \operatorname{Lie}(A) ;\left(\tau_{*}, \operatorname{id}_{\operatorname{Lie}(A)}\right)(\xi) \in \mathbb{C} \cdot v\right\} .
$$

Then we have $q_{k}^{*} V_{k}^{\dagger}=V_{k}$.

Let $T_{M_{k} / S_{[k]}} \subset T_{M_{k}}$ be the relative tangent bundle with respect to the second projection $q_{k}: M_{k} \rightarrow S_{[k]}$. We define $M_{k}^{o} \subset M_{k}$ by

$$
M_{k}^{o}=\left\{x \in M_{k} ;\left(V_{k} \cap T_{M_{k} / S_{[k]}}\right)_{x} \neq\{0\}\right\} .
$$

We set $S_{[k]}^{o}=q_{k}\left(M_{k}^{o}\right)$. Then $S_{[k]}^{o} \subset S_{[k]}$ and $M_{k}^{o}=A \times S_{[k]}^{o}$.

We claim that

$$
S_{[k]}^{o}=S \times \mathbb{P}(\operatorname{Lie}(A))
$$

for all $k \geq 1$. We prove this. For $k \geq 1$, let $\pi_{k}: M_{k} \rightarrow M_{k-1}$ be the projection. Let $(x,[v]) \in M_{k}$, where $x \in M_{k-1}$ and $v \in\left(V_{k-1}\right)_{x}$ with $v \neq 0$. Let $\left(\pi_{k}\right)_{*}:\left(T M_{k}\right)_{(x,[v])} \rightarrow$ $\left(T M_{k-1}\right)_{x}$ be the induced map. Then we have $\left(V_{k}\right)_{(x,[v])}=\left(\left(\pi_{k}\right)_{*}\right)^{-1}(\mathbb{C} v)$. The map $\left(\pi_{k}\right)_{*}$ induces an isomorphism $\left(T_{M_{k} / S_{[k]}}\right)_{(x,[v])} \rightarrow\left(T_{M_{k-1} / S_{[k-1]}}\right)_{x}$. Hence $\left(\pi_{k}\right)_{*}$ induces an isomorphism

$$
\left(V_{k} \cap T_{M_{k} / S_{[k]}}\right)_{(x,[v])} \stackrel{\sim}{\rightarrow}(\mathbb{C} v) \cap\left(T_{M_{k-1} / S_{[k-1]}}\right)_{x} .
$$

This shows that $(x,[v]) \in M_{k}^{o}$ if and only if $x \in M_{k-1}^{o}$ and $v \in\left(V_{k-1} \cap T_{M_{k-1} / S_{[k-1]}}\right)_{x}$. Hence we have

$$
M_{k}^{o}=\mathbb{P}\left(\left.\left(V_{k-1} \cap T_{M_{k-1} / S_{[k-1]}}\right)\right|_{M_{k-1}^{o}}\right) .
$$

We first check (4.1) for $k=1$. We have $M_{1}^{o}=\mathbb{P}\left(T_{A \times S / S}\right)=A \times S \times \mathbb{P}(\operatorname{Lie}(A))$. This shows (4.1) for $k=1$. Next we assume that (4.1) is true for $k$, where $k \geq 1$. 
Then by (4.2), the restriction $\left.\left(V_{k} \cap T_{M_{k} / S_{[k]}}\right)\right|_{M_{k}^{o}}$ is a rank one vector bundle. Hence $M_{k+1}^{o}=\mathbb{P}\left(\left.\left(V_{k} \cap T_{M_{k} / S_{[k]}}\right)\right|_{M_{k}^{o}}\right)=M_{k}^{o}$. Thus (4.1) also holds for $k+1$. By the induction, we have proved (4.1).

For $v \in \operatorname{Lie}(A)-\{0\}$, let $\varphi_{v}: \mathbb{C} \rightarrow A$ be a one parameter subgroup such that $\varphi_{v}^{\prime}(0)=$ $v$. For $(a, s) \in A \times S$, let $\varphi_{v,(a, s)}: \mathbb{C} \rightarrow A \times S$ be defined by $\varphi_{v,(a, s)}(z)=\left(a+\varphi_{v}(z), s\right)$. Then we have

$$
\left(\varphi_{v,(a, s)}\right)_{[k]}(z)=\left(a+\varphi_{v}(z), s,[v]\right) \in A \times S_{[k]}^{o} .
$$

Now let $X \subset A \times S$ be a closed subscheme. Let $p_{k}: X_{k} \rightarrow S_{[k]}$ be the composite of the closed immersion $X_{k} \hookrightarrow A \times S_{[k]}$ and the second projection $A \times S_{[k]} \rightarrow S_{[k]}$. We fix some projective embedding $A \subset \mathbb{P}^{n}$. We define a Zariski closed subset $T_{[k]} \subset S_{[k]}$ as in (3.1) from the projective morphism $p_{k}: X_{k} \rightarrow S_{[k]}$. Then $T_{[k]} \subset p_{k}\left(X_{k}\right)$.

Lemma 4.2. Assume that $X_{s} \neq A$ for all $s \in S$ and that $X_{k} \subset A \times S_{[k]}$ is nonempty for all $k \geq 0$. Then there exists a positive integer $\nu>0$ with the following property: Let $T$ be a connected component of $T_{[\nu]}$ such that $T \cap S_{[\nu]}^{o} \neq \emptyset$. Then there exists a proper abelian subvariety $B \varsubsetneqq A$ such that $T \cap S_{[\nu]}^{o} \subset S \times \mathbb{P}(\operatorname{Lie}(B))$.

REMARK 4.3. The proof shows that there exists an integer $\nu_{0}>0$ such that all integers $\nu \geq \nu_{0}$ satisfy the property of Lemma 4.2 .

Proof of Lemma 4.2.

Step 1: We first find $\nu$ in the statement. The restrictions of the projection maps $\pi_{k}: M_{k} \rightarrow M_{k-1}$ induce isomorphisms $M_{k}^{o} \rightarrow M_{k-1}^{o}$. We consider $\operatorname{supp}\left(X_{k} \cap M_{k}^{o}\right)$ as Zariski closed subsets of $M_{k}^{o}=A \times S \times \mathbb{P}(\operatorname{Lie}(A))$, which form a nested sequence

$$
A \times S \times \mathbb{P}(\operatorname{Lie}(A)) \supset \operatorname{supp}\left(X_{1} \cap M_{1}^{o}\right) \supset \operatorname{supp}\left(X_{2} \cap M_{2}^{o}\right) \supset \operatorname{supp}\left(X_{3} \cap M_{3}^{o}\right) \supset \cdots .
$$

By the Noetherian property, there exists an integer $\nu$ such that

$$
\operatorname{supp}\left(X_{\nu} \cap M_{\nu}^{o}\right)=\operatorname{supp}\left(X_{\nu+1} \cap M_{\nu+1}^{o}\right)=\operatorname{supp}\left(X_{\nu+2} \cap M_{\nu+2}^{o}\right)=\cdots .
$$

We fix this $\nu$.

Step 2: For $v \in \operatorname{Lie}(A)-\{0\}$, let $B_{v} \subset A$ be the Zariski closure of $\varphi_{v}(\mathbb{C})$, where $\varphi_{v}: \mathbb{C} \rightarrow A$ is the one parameter subgroup. Then $B_{v}$ is a positive dimensional abelian subvariety of $A$. Let $(s,[v]) \in p_{\nu}\left(X_{\nu}\right) \cap S_{[\nu]}^{o} \subset S \times \mathbb{P}(\operatorname{Lie}(A))$. We claim that

$$
B_{v} \subset \operatorname{St}\left(\operatorname{supp} p_{\nu}^{-1}((s,[v]))\right)
$$

where $\operatorname{St}\left(\operatorname{supp} p_{\nu}^{-1}((s,[v]))\right)$ is the stabilizer of $\operatorname{supp} p_{\nu}^{-1}((s,[v])) \subset A$. Indeed, suppose $a \in \operatorname{supp} p_{\nu}^{-1}((s,[v]))$. Then $(a, s) \in X \subset A \times S$. By (4.3), the definition of $\nu$ yields that $\left(\varphi_{v,(a, s)}\right)_{[k]}(0)=(a, s,[v]) \in X_{k}$ for all $k \geq 0$. By Taylor series, we have $\varphi_{v,(a, s)}(\mathbb{C}) \subset X$. Hence $a+B_{v} \subset X_{s}$. Now for arbitrary $x \in a+B_{v}$, we have $\varphi_{v,(x, s)}(\mathbb{C}) \subset X$. This shows $\left(\varphi_{v,(x, s)}\right)_{[\nu]}(0)=(x, s,[v]) \in X_{\nu}$. Hence $x \in \operatorname{supp} p_{\nu}^{-1}((s,[v]))$. Thus we have $a+B_{v} \subset \operatorname{supp} p_{\nu}^{-1}((s,[v]))$ for all $a \in \operatorname{supp} p_{\nu}^{-1}((s,[v]))$. This shows (4.4).

Step 3: We consider a general situation. Let $\Sigma$ be a variety (cf. Remark 1.6) and let 
$Z \subset A \times \Sigma$ be a non-empty, closed subscheme such that the projection $\psi: Z \rightarrow \Sigma$ is flat. Then the dimension of $Z_{s}$ is independent of $s \in \Sigma$ (cf. [12, III, Corollary 9.10]). We set this dimension $d$. For each $s \in \Sigma$, let $\left(Z_{s}\right)^{\prime}$ be the union of all $d$-dimensional irreducible components of $\operatorname{supp} Z_{s}$. Then $\left(Z_{s}\right)^{\prime}$ is a Zariski closed subset of $A$. Let $\operatorname{St}^{0}\left(\left(Z_{s}\right)^{\prime}\right)$ be the connected component of $\operatorname{St}\left(\left(Z_{s}\right)^{\prime}\right)$ which contains the identity element $0 \in A$. Then $\mathrm{St}^{0}\left(\left(Z_{s}\right)^{\prime}\right)$ is a (possibly trivial) abelian subvariety of $A$. We prove the following

Claim. $\operatorname{St}^{0}\left(\left(Z_{s}\right)^{\prime}\right)$ is independent of $s \in \Sigma$.

When $\operatorname{dim} \Sigma=0$, this claim is trivial. In the following, we assume $\operatorname{dim} \Sigma>0$. We fix $s_{0} \in \Sigma$ arbitrary. Let $D=\operatorname{St}^{0}\left(\left(Z_{s_{0}}\right)^{\prime}\right)$. We show that $D=\operatorname{St}^{0}\left(\left(Z_{s}\right)^{\prime}\right)$ for all $s \in \Sigma$. By taking a chain of irreducible curves connecting $s_{0}$ and $s$ (cf. [24, p.56]) and considering the normalizations of these curves, we may assume that $\Sigma$ is a smooth curve. Thus $\operatorname{dim} Z=d+1$.

It is sufficient to show that $D \subset \operatorname{St}^{0}\left(\left(Z_{s}\right)^{\prime}\right)$ for all $s \in \Sigma$. Let $Y_{1}, \ldots, Y_{k}$ be the irreducible components of $\operatorname{supp} Z$. We assume that $\operatorname{dim} Y_{j}=d+1$ for $1 \leq j \leq l$ and $\operatorname{dim} Y_{j}<d+1$ for $l+1 \leq j \leq k$. Let $\left(Y_{j}\right)_{s}$ be the fiber of the restriction map $\left.\psi\right|_{Y_{j}}: Y_{j} \rightarrow \Sigma$ over $s \in \Sigma$. Since $\psi: Z \rightarrow \Sigma$ is flat, we have $\psi\left(Y_{j}\right)=\Sigma$ for every $1 \leq j \leq k$ (cf. [12, III, Proposition 9.7]). Hence for every $1 \leq j \leq k$ and $s \in \Sigma$, all irreducible components of $\left(Y_{j}\right)_{s}$ have the same dimension $\operatorname{dim} Y_{j}-1$. Thus for every $s \in \Sigma$, we have

$$
D \subset \operatorname{St}^{0}\left(\left(Z_{s}\right)^{\prime}\right) \Longleftrightarrow D \subset \operatorname{St}^{0}\left(\operatorname{supp}\left(Y_{j}\right)_{s}\right) \text { for all } 1 \leq j \leq l .
$$

Now we fix $1 \leq j \leq l$, and prove $D \subset \mathrm{St}^{0}\left(\operatorname{supp}\left(Y_{j}\right)_{s}\right)$ for all $s \in \Sigma$. Indeed otherwise, denoting by $W_{j} \subset(A / D) \times \Sigma$ the image of $Y_{j}$ under the projection $A \times \Sigma \rightarrow(A / D) \times \Sigma$, we have

$$
\operatorname{dim} W_{j}+\operatorname{dim} D>\operatorname{dim} Y_{j}=d+1 .
$$

On the other hand, denoting by $\phi_{j}: W_{j} \rightarrow \Sigma$ the induced map, we have

$$
\operatorname{dim} \phi_{j}^{-1}\left(s_{0}\right)=\operatorname{dim}\left(Y_{j}\right)_{s_{0}}-\operatorname{dim} D=d-\operatorname{dim} D .
$$

Hence we have

$$
\operatorname{dim} W_{j}-1>d-\operatorname{dim} D=\operatorname{dim} \phi_{j}^{-1}\left(s_{0}\right) .
$$

This contradicts to $\operatorname{dim} \phi_{j}^{-1}\left(s_{0}\right)=\operatorname{dim} W_{j}-1$. Thus $D \subset \operatorname{St}^{0}\left(\operatorname{supp}\left(Y_{j}\right)_{s}\right)$ for all $s \in \Sigma$. Hence $D \subset \mathrm{St}^{0}\left(\left(Z_{s}\right)^{\prime}\right)$ for all $s \in \Sigma$. This conclude the proof of the claim.

Step 4: We return to the proof of our lemma. Let $T$ be a connected component of $T_{[\nu]}$ such that $T \cap S_{[\nu]}^{o} \neq \emptyset$. Let $T^{\prime}$ be an irreducible component of $T$. Then $T^{\prime}$ is irreducible and reduced, and hence a variety. Let $\left(p_{\nu}\right)_{T^{\prime}}:\left(X_{\nu}\right)_{T^{\prime}} \rightarrow T^{\prime}$ be the base change of $p_{\nu}: X_{\nu} \rightarrow S_{[\nu]}$. By the construction of $T_{[\nu]}$, the Hilbert polynomials of the fibers of $\left(p_{\nu}\right)_{T^{\prime}}$ are all the same. Hence $\left(p_{\nu}\right)_{T^{\prime}}$ is flat (cf. [12, III, Theorem 9.9]). Note that $\left(X_{\nu}\right)_{T^{\prime}} \neq \emptyset$, for $X_{\nu} \neq \emptyset$. By the claim above, $\mathrm{St}^{0}\left(\left(\left(X_{\nu}\right)_{t}\right)^{\prime}\right)$ is independent of $t \in T^{\prime}$. Since $T$ is connected, $\operatorname{St}^{0}\left(\left(\left(X_{\nu}\right)_{t}\right)^{\prime}\right)$ is independent of $t \in T$. We denote this abelian subvariety by $B$. Then by the assumption that $X_{s} \neq A$ for all $s \in S$ and $X_{\nu} \neq \emptyset$, we 
have $\left(\left(X_{\nu}\right)_{t}\right)^{\prime} \neq A$ and $\left(\left(X_{\nu}\right)_{t}\right)^{\prime} \neq \emptyset$ for all $t \in T$. Thus $B \neq A$. Now let $(s,[v]) \in T \cap S_{[\nu]}^{o}$. Then by (4.4), we have

$$
B_{v} \subset \mathrm{St}^{0}\left(\operatorname{supp}\left(\left(X_{\nu}\right)_{(s,[v])}\right)\right) \subset \mathrm{St}^{0}\left(\left(\left(X_{\nu}\right)_{(s,[v])}\right)^{\prime}\right)=B .
$$

Hence $[v] \in \mathbb{P}(\operatorname{Lie}(B))$, thus $(s,[v]) \in S \times \mathbb{P}(\operatorname{Lie}(B))$. This shows $T \cap S_{[\nu]}^{o} \subset S \times \mathbb{P}(\operatorname{Lie}(B))$, which completes the proof of Lemma 4.2 .

We discuss a consequence of Lemma 4.2, which is needed in the proof of Proposition 2.3. For $v \in T\left(A \times S_{[\nu]}\right)$, we denote by $v_{A} \in T A$ (resp. $\left.v_{S_{[\nu]}} \in T S_{[\nu]}\right)$ the image of $v$ under the induced map $T\left(A \times S_{[\nu]}\right) \rightarrow T A\left(\operatorname{resp} . T\left(A \times S_{[\nu]}\right) \rightarrow T S_{[\nu]}\right)$.

Lemma 4.4. Let $X \subset A \times S$ be the same as in Lemma 4.2 and let $\nu>0$ be a positive integer which satisfies the property described in Lemma 4.2. Let $T$ be a connected component of $T_{[\nu]} \subset S_{[\nu]}$. Then there exists $B \in \Sigma(A)$ with the following property: Let $\varepsilon>0$. Let $\omega_{A}$ be a positive invariant $(1,1)$-form on $A$ and let $\omega_{S_{[\nu]}}$ be a smooth positive $(1,1)$-form on $S_{[\nu]}$. Let $\omega_{A / B}$ be a positive invariant $(1,1)$-form on $A / B$. Then there exist an open subset $U \subset S_{[\nu]}$ with $T \subset U$ and a positive constant $\rho>0$ such that

$$
\left|\left(\varpi_{B}\right)_{*}\left(\left(v_{x}\right)_{A}\right)\right|_{\omega_{A / B}}^{2} \leq \varepsilon\left|\left(v_{x}\right)_{A}\right|_{\omega_{A}}^{2}+\rho\left|\left(v_{x}\right)_{S_{[\nu]}}\right|_{\omega_{S_{[\nu]}}}^{2}
$$

for all $x \in A \times U$ and all $v_{x} \in\left(V_{\nu}\right)_{x} \subset T_{x}\left(A \times S_{[\nu]}\right)$, where $\left(\varpi_{B}\right)_{*}: T A \rightarrow T(A / B)$ is the map induced from the quotient map $\varpi_{B}: A \rightarrow A / B$.

Proof. $\quad$ For each open set $W \Subset S_{[\nu]} \backslash S_{[\nu]}^{o}$, there exists a positive constant $\gamma_{W}>0$ such that

$$
\left|\left(v_{(a, s)}\right){ }_{A}\right|_{\omega_{A}} \leq \gamma_{W}\left|\left(v_{(a, s)}\right)_{S_{[\nu]}}\right|_{\omega_{S_{[\nu]}}}
$$

for all $(a, s) \in A \times W$ and all $v_{(a, s)} \in\left(V_{\nu}\right)_{(a, s)} \subset T_{(a, s)}\left(A \times S_{[\nu]}\right)$. This follows from the fact that the composition of the natural maps

$$
\left(V_{\nu}\right)_{(a, s)} \hookrightarrow T_{(a, s)}\left(A \times S_{[\nu]}\right) \rightarrow T_{s}\left(S_{[\nu]}\right)
$$

is injective for all $(a, s) \in A \times\left(S_{[\nu]} \backslash S_{[\nu]}^{o}\right)$. Hence if $T \cap S_{[\nu]}^{o}=\emptyset$, we take $B=\{0\}$ and $U \subset S_{[\nu]}$ such that $T \subset U$ and $U \Subset S_{[\nu]} \backslash S_{[\nu]}^{o}$.

We assume that $T \cap S_{[\nu]}^{o} \neq \emptyset$. By Lemma 4.2, there exists $B \in \Sigma(A)$ such that $T \cap S_{[\nu]}^{o} \subset S \times \mathbb{P}(\operatorname{Lie} B)$. We claim that there exists an open subset $U_{1} \subset S_{[\nu]}$ with $S \times \mathbb{P}($ Lie $B) \subset U_{1}$ such that

$$
\left|\left(\varpi_{B}\right)_{*}\left(\left(v_{x}\right)_{A}\right)\right|_{\omega_{A / B}}^{2} \leq \varepsilon\left|v_{x}\right|_{\omega_{A \times S_{[\nu]}}}^{2}=\varepsilon\left(\left|\left(v_{x}\right)_{A}\right|_{\omega_{A}}^{2}+\left|\left(v_{x}\right)_{S_{[\nu]}}\right|_{\omega_{S}[\nu]}^{2}\right)
$$

for all $x \in A \times U_{1}$ and all $v_{x} \in\left(V_{\nu}\right)_{x} \subset T_{x}\left(A \times S_{[\nu]}\right)$. We prove this. Let $x \in A \times$ $(S \times \mathbb{P}(\operatorname{Lie} B)) \subset A \times S_{[\nu]}^{o}$ and $v_{x} \in\left(V_{\nu}\right)_{x}$. Then by the construction of $S_{[\nu]}^{o}$, we have $\left(\pi_{\nu}\right)_{*}\left(v_{x}\right) \in T_{A \times S_{[\nu-1]} / S_{[\nu-1]}}$ and

$$
\left(\pi_{\nu}\right)_{*}\left(v_{x}\right) \in \operatorname{Lie} B \subset \operatorname{Lie} A=\left(T_{A \times S_{[\nu-1]} / S_{[\nu-1]}}\right)_{\pi_{\nu}(x)} .
$$


Hence $\left|\left(\varpi_{B}\right)_{*}\left(\left(v_{x}\right)_{A}\right)\right|_{\omega_{A / B}}=0$ for all $x \in A \times(S \times \mathbb{P}($ Lie $B))$ and all $v_{x} \in\left(V_{\nu}\right)_{x}$. We define a compact set $K \subset V_{\nu}$ by

$$
K=\left\{v \in V_{\nu} ;|v|_{\omega_{A \times S}[\nu]}=1\right\} .
$$

For $x \in A \times S_{[\nu]}$, we set $K_{x}=K \cap\left(V_{\nu}\right)_{x}$. We set

$$
K^{\prime}=\left\{v \in K ;\left|\left(\varpi_{B}\right)_{*}\left(v_{A}\right)\right|_{\omega_{A / B}} \geq \sqrt{\varepsilon}\right\} .
$$

Then $K^{\prime} \subset K$ is compact and

$$
K^{\prime} \cap \bigcup_{x \in A \times(S \times \mathbb{P}(\operatorname{Lie} B))} K_{x}=\emptyset .
$$

Hence there exists an open set $U_{1} \subset S_{[\nu]}$ such that $S \times \mathbb{P}(\operatorname{Lie} B) \subset U_{1}$ and

$$
\left|\left(\varpi_{B}\right)_{*}\left(\left(v_{x}\right)_{A}\right)\right|_{\omega_{A / B}}<\sqrt{\varepsilon}
$$

for all $x \in A \times U_{1}$ and $v_{x} \in K$. Hence we have (4.6) for all $x \in A \times U_{1}$ and all $v_{x} \in\left(V_{\nu}\right)_{x}$.

Now we take $U_{2} \Subset S_{[\nu]} \backslash S_{[\nu]}^{o}$ such that $T \subset U_{1} \cup U_{2}$. We set $U=U_{1} \cup U_{2}$. If $x \in A \times U_{2}$ and $v_{x} \in\left(V_{\nu}\right)_{x}$, then we have

$$
\left|\left(\varpi_{B}\right)_{*}\left(\left(v_{x}\right)_{A}\right)\right|_{\omega_{A / B}} \leq c\left|\left(v_{x}\right)_{A}\right|_{\omega_{A}} \leq c \gamma_{U_{2}}\left|\left(v_{x}\right)_{S_{[\nu]}}\right|_{\omega_{S_{[\nu]}}},
$$

where $c$ is a positive constant such that $\varpi_{B}^{*} \omega_{A / B} \leq c^{2} \omega_{A}$. Hence (4.5) is valid for $\rho=\max \left\{\varepsilon, c^{2} \gamma_{U_{2}}^{2}\right\}$.

\section{Tautological inequality.}

We introduce a variant of the tautological inequality. For entire curves, this estimate is due to Kobayashi [18] and McQuillan [23]. See also [3], [33], [35].

Let $X$ be a smooth projective variety. Let $\omega_{X}$ be a smooth, positive $(1,1)$-form on $X$. Then $\omega_{X}$ naturally induces a Hermitian metric on the tautological line bundle $\mathcal{O}_{P T X}(1)$ on $P T X$. Let $\omega_{\mathcal{O}_{P T X}(1)}$ be the associated curvature form for the tautological line bundle $\mathcal{O}_{P T X}(1)$ on $P T X$.

Lemma 5.1. Let $X, \omega_{X}, \omega_{\mathcal{O}_{P T X}(1)}$ be as above. Let $0<s<1, \varepsilon>0$ and $\delta>0$. Then there exists a positive constant $\mu>0$ such that for every non-constant holomorphic map $f: \mathbb{D} \rightarrow X$, the estimate

$$
\int_{s}^{r} \frac{d t}{t} \int_{\mathbb{D}(t)}\left(f_{[1]}\right)^{*} \omega_{\mathcal{O}_{P T X}(1)} \leq \varepsilon \int_{s}^{r} d t \int_{\mathbb{D}(t)} f^{*} \omega_{X}+\int_{0}^{2 \pi} \log \frac{1}{\left|f^{\prime}\left(s e^{i \theta}\right)\right|_{\omega_{X}}} \frac{d \theta}{2 \pi}+\mu
$$

holds for all $r \in(s, 1)$ outside some exceptional set $E \subset(s, 1)$ with the linear measure $|E|<\delta$.

Proof. We follow the argument in $[\mathbf{3}]$. The metric $\omega_{X}$ defines a Hermitian metric $|\cdot|_{\omega_{X}}$ on $\mathcal{O}_{P T X}(-1)$, whose curvature form is $-\omega_{\mathcal{O}_{P T X}(1)}$. By the Poincaré-Lelong 
formula, we have

$$
-\left(f_{[1]}\right)^{*} \omega_{\mathcal{O}_{P T X}(1)}=\left[\left(f^{\prime}\right)^{*} F\right]-2 d d^{c} \log \left|f^{\prime}\right|_{\omega_{X}}
$$

as currents on $\mathbb{D}$, where $F$ is the zero section of $\mathcal{O}_{P T X}(-1)$. By the Jensen formula, we have

$$
\int_{s}^{r} \frac{d t}{t} \int_{\mathbb{D}(t)}\left(f_{[1]}\right)^{*} \omega_{\mathcal{O}_{P T X}(1)} \leq \int_{0}^{2 \pi} \log \left|f^{\prime}\left(r e^{i \theta}\right)\right|_{\omega_{X}} \frac{d \theta}{2 \pi}-\int_{0}^{2 \pi} \log \left|f^{\prime}\left(s e^{i \theta}\right)\right|_{\omega_{X}} \frac{d \theta}{2 \pi} .
$$

Using concavity of log, we have

$$
\int_{0}^{2 \pi} \log \left|f^{\prime}\left(r e^{i \theta}\right)\right|_{\omega_{X}} \frac{d \theta}{2 \pi} \leq \frac{1}{2} \log \int_{0}^{2 \pi}\left|f^{\prime}\left(r e^{i \theta}\right)\right|_{\omega_{X}}^{2} \frac{d \theta}{2 \pi} .
$$

We set

$$
T(r)=\int_{s}^{r} d t \int_{\mathbb{D}(t)} f^{*} \omega_{X}
$$

Then we have

$$
\frac{1}{2 \pi r} \frac{d^{2}}{d r^{2}} T(r)=\int_{0}^{2 \pi}\left|f^{\prime}\left(r e^{i \theta}\right)\right|_{\omega_{X}}^{2} \frac{d \theta}{2 \pi}
$$

Hence

$$
\int_{0}^{2 \pi} \log \left|f^{\prime}\left(r e^{i \theta}\right)\right|_{\omega_{X}} \frac{d \theta}{2 \pi} \leq \frac{1}{2} \log \left(\frac{1}{2 \pi r} \frac{d^{2}}{d r^{2}} T(r)\right) .
$$

Hence for $r>s$, we have

$$
\int_{0}^{2 \pi} \log \left|f^{\prime}\left(r e^{i \theta}\right)\right|_{\omega_{X}} \frac{d \theta}{2 \pi} \leq \frac{1}{2} \log \left(\frac{d^{2}}{d r^{2}} T(r)\right)-\frac{\log 2 \pi s}{2} .
$$

Now we apply Lemma 5.2 below twice. We have

$$
\begin{aligned}
\log \left(\frac{d^{2}}{d r^{2}} T(r)\right) & \leq \log \left(\frac{4}{\delta} \max \left\{1,\left(T^{\prime}(r)\right)^{2}\right\}\right) \\
& \leq \log \left(\frac{4}{\delta} \max \left\{1,\left(\frac{4}{\delta} \max \left\{1, T(r)^{2}\right\}\right)^{2}\right\}\right) \\
& =\log \left(\frac{4^{3}}{\delta^{3}} \max \left\{1, T(r)^{4}\right\}\right)
\end{aligned}
$$

for $r \in(s, 1)$ outside some exceptional set $E$ with $|E|<\delta$. Hence

$$
\int_{s}^{r} \frac{d t}{t} \int_{\mathbb{D}(t)}\left(f_{[1]}\right)^{*} \omega_{\mathcal{O}_{P T X}(1)} \leq 2 \log ^{+} T(r)+\int_{0}^{2 \pi} \log \frac{1}{\left|f^{\prime}\left(s e^{i \theta}\right)\right|_{\omega_{X}}} \frac{d \theta}{2 \pi}-\frac{\log 2 \pi s}{2}+\frac{3}{2} \log \frac{4}{\delta}
$$


for $r \in(s, 1)$ outside $E$. We take a positive constant $\mu^{\prime}>0$ such that $2 \log ^{+} x<\varepsilon x+\mu^{\prime}$ for $x \geq 0$. Then we obtain our estimate.

LEMMA 5.2. Let $g$ be a continuously differentiable, increasing function on $[s, 1)$ with $g(s) \geq 0$. Let $\delta>0$. Then we have

$$
g^{\prime}(r) \leq \frac{2}{\delta} \max \left\{1, g(r)^{2}\right\}
$$

for all $r \in(s, 1)$ outside a set $E_{\delta}$ with $\left|E_{\delta}\right|<\delta$.

Proof. Set

$$
E_{\delta}=\left\{r \in(s, 1) ; g^{\prime}(r)>\frac{2}{\delta} \max \left\{1, g(r)^{2}\right\}\right\}
$$

If $E_{\delta}=\emptyset$, then our assertion is trivial. Suppose $E_{\delta} \neq \emptyset$. We have

$$
\left|E_{\delta}\right|<\frac{\delta}{2} \int_{E_{\delta}} \frac{g^{\prime}(r)}{\max \left\{1, g(r)^{2}\right\}} d r \leq \frac{\delta}{2} \int_{s}^{1} \frac{g^{\prime}(r)}{\max \left\{1, g(r)^{2}\right\}} d r .
$$

We have the following three cases.

Case 1: $g(r) \geq 1$ for all $r \in[s, 1)$. Then we have

$$
\int_{s}^{1} \frac{g^{\prime}(r)}{\max \left\{1, g(r)^{2}\right\}} d r=\int_{s}^{1} \frac{g^{\prime}(r)}{g(r)^{2}} d r=\lim _{t \rightarrow 1-0}\left[\frac{-1}{g(r)}\right]_{s}^{t} \leq 1 .
$$

Case 2: $g(r) \leq 1$ for all $r \in[s, 1)$. Then we have

$$
\int_{s}^{1} \frac{g^{\prime}(r)}{\max \left\{1, g(r)^{2}\right\}} d r=\int_{s}^{1} g^{\prime}(r) d r \leq 1 .
$$

Case 3: Otherwise, we have $g(s)<1$ and $\lim _{r \rightarrow 1-0} g(r)>1$. We set $\kappa=\sup \{r \in$ $[s, 1) ; g(r) \leq 1\}$. Then we have $s<\kappa<1$ and $g(\kappa)=1$. Hence we have

$$
\int_{s}^{1} \frac{g^{\prime}(r)}{\max \left\{1, g(r)^{2}\right\}} d r=\int_{s}^{\kappa} g^{\prime}(r) d r+\int_{\kappa}^{1} \frac{g^{\prime}(r)}{g(r)^{2}} d r \leq 1+\lim _{t \rightarrow 1-0}\left[\frac{-1}{g(r)}\right]_{\kappa}^{t} \leq 2 .
$$

Thus in all cases, we have proved $\left|E_{\delta}\right|<\delta$.

Now let $A$ be an abelian variety and let $S$ be a smooth projective variety. Then by Lemma 4.1, there exists a smooth projective variety $S_{[k]}$ such that $(A \times S)_{k}=A \times S_{[k]}$.

Lemma 5.3. Let $\omega_{A}$ be a positive invariant $(1,1)$-form on $A$ and let $\omega_{S}$ be a smooth, positive $(1,1)$-form on $S$. For $k \in \mathbb{Z}_{\geq 0}$, let $\omega_{S_{[k]}}$ be a smooth, positive $(1,1)$-form on $S_{[k]}$. Let $0<s<1, \varepsilon>0, \delta>0$. Then there exist positive constants $\mu_{1}, \mu_{2}, \mu_{3}$ such that for every non-constant holomorphic map $f: \mathbb{D} \rightarrow A \times S$, the estimate 


$$
\begin{aligned}
\int_{s}^{r} d t \int_{\mathbb{D}(t)}\left(\left(f_{[k]}\right)_{S_{[k]}}\right)^{*} \omega_{S_{[k]}} \leq & \varepsilon \int_{s}^{r} d t \int_{\mathbb{D}(t)} f_{A}^{*} \omega_{A}+\mu_{1} \int_{s}^{r} d t \int_{\mathbb{D}(t)} f_{S}^{*} \omega_{S} \\
& +\mu_{2} \max \left\{0, \int_{0}^{2 \pi} \log \frac{1}{\left|f^{\prime}\left(s e^{i \theta}\right)\right|_{\omega_{A \times S}}} \frac{d \theta}{2 \pi}\right\}+\mu_{3}
\end{aligned}
$$

holds for $r \in(s, 1)$ outside some exceptional set of linear measure less than $\delta$.

Proof. We prove by the induction on $k$. When $k=0$, our assertion is trivial. Suppose that our estimate is valid for $k-1$, where $k \geq 1$. Then $P\left(V_{k-1}\right)=A \times S_{[k]}$ for the vector subbundle $V_{k-1} \subset T\left(A \times S_{[k-1]}\right)$. Let $q: A \times S_{[k-1]} \rightarrow S_{[k-1]}$ be the second projection. By Lemma 4.1, there exists a vector bundle $V_{k-1}^{\dagger}$ on $S_{[k-1]}$ such that $q^{*} V_{k-1}^{\dagger}=V_{k-1}$. Then we have $P\left(V_{k-1}^{\dagger}\right)=S_{[k]}$. We note that $\omega_{A}$ and $\omega_{S_{[k-1]}}$ induce Hermitian metrics on $V_{k-1}^{\dagger}$ and $V_{k-1}$, hence on the tautological bundles $\mathcal{O}_{P V_{k-1}^{\dagger}}(1)$ and $\mathcal{O}_{P V_{k-1}}(1)$. We denote by $\omega_{\mathcal{O}_{P V_{k-1}^{\dagger}}(1)}$ and $\omega_{\mathcal{O}_{P V_{k-1}}(1)}$ the associated curvature forms on $S_{[k]}$ and $A \times S_{[k]}$, respectively.

Let $\tau: S_{[k]} \rightarrow S_{[k-1]}$ be the induced map. There exist positive constants $\alpha_{1}, \alpha_{2}$ such that

$$
\omega_{S_{[k]}} \leq \alpha_{1} \omega_{\mathcal{O}_{P V_{k-1}^{\dagger}}(1)}+\alpha_{2} \tau^{*} \omega_{S_{[k-1]}}
$$

on $S_{[k]}$. By Lemma 5.1, we get

$$
\begin{aligned}
& \int_{s}^{r} \frac{d t}{t} \int_{\mathbb{D}(t)}\left(f_{[k]}\right)^{*} \omega_{\mathcal{O}_{P V_{k-1}}(1)} \\
& \quad \leq \frac{\varepsilon}{2 \alpha_{1}} \int_{s}^{r} d t \int_{\mathbb{D}(t)}\left(f_{[k-1]}\right)^{*} \omega_{A \times S_{[k-1]}}+\int_{0}^{2 \pi} \log \frac{1}{\left|f^{\prime}\left(s e^{i \theta}\right)\right|_{\omega_{A \times S}}} \frac{d \theta}{2 \pi}+\mu
\end{aligned}
$$

for $r \in(s, 1)$ outside some exceptional set $E_{1}$ with $\left|E_{1}\right|<\delta / 2$. Hence we get

$$
\begin{aligned}
& \int_{s}^{r} d t \int_{\mathbb{D}(t)}\left(\left(f_{[k]}\right)_{S_{[k]}}\right)^{*} \omega_{S_{[k]}} \\
& \leq \int_{s}^{r} \frac{d t}{t} \int_{\mathbb{D}(t)}\left(\left(f_{[k]}\right)_{S_{[k]}}\right)^{*} \omega_{S_{[k]}} \\
& \leq \alpha_{1} \int_{s}^{r} \frac{d t}{t} \int_{\mathbb{D}(t)}\left(f_{[k]}\right)^{*} \omega_{\mathcal{O}_{P V_{k-1}}(1)}+\frac{\alpha_{2}}{s} \int_{s}^{r} d t \int_{\mathbb{D}(t)}\left(\left(f_{[k-1]}\right)_{S_{[k-1]}}\right)^{*} \omega_{S_{[k-1]}} \\
& \leq \frac{\varepsilon}{2} \int_{s}^{r} d t \int_{\mathbb{D}(t)}\left(f_{[k-1]}\right)^{*} \omega_{A \times S_{[k-1]}}+\frac{\alpha_{2}}{s} \int_{s}^{r} d t \int_{\mathbb{D}(t)}\left(\left(f_{[k-1]}\right)_{S_{[k-1]}}\right)^{*} \omega_{S_{[k-1]}} \\
&+\alpha_{1} \int_{0}^{2 \pi} \log \frac{1}{\left|f^{\prime}\left(s e^{i \theta}\right)\right|_{\omega_{A \times S}} \frac{d \theta}{2 \pi}+\alpha_{1} \mu} \\
& \leq \frac{\varepsilon}{2} \int_{s}^{r} d t \int_{\mathbb{D}(t)} f_{A}^{*} \omega_{A}+\left(\frac{\varepsilon}{2}+\frac{\alpha_{2}}{s}\right) \int_{s}^{r} d t \int_{\mathbb{D}(t)}\left(\left(f_{[k-1]}\right)_{S_{[k-1]}}\right)^{*} \omega_{S_{[k-1]}} \\
&+\alpha_{1} \max \left\{0, \int_{0}^{2 \pi} \log \frac{1}{\left|f^{\prime}\left(s e^{i \theta}\right)\right|_{\omega_{A \times S}}} \frac{d \theta}{2 \pi}\right\}+\alpha_{1} \mu
\end{aligned}
$$


for $r \in(s, 1)$ outside $E_{1}$. Now by the induction hypothesis, we have

$$
\begin{aligned}
\int_{s}^{r} d t \int_{\mathbb{D}(t)}\left(\left(f_{[k-1]}\right)_{S_{[k-1]}}\right)^{*} \omega_{S_{[k-1]}} \leq & \frac{\varepsilon s}{2 \alpha_{2}+\varepsilon s} \int_{s}^{r} d t \int_{\mathbb{D}(t)} f_{A}^{*} \omega_{A}+\mu_{1}^{\prime} \int_{s}^{r} d t \int_{\mathbb{D}(t)} f_{S}^{*} \omega_{S} \\
& +\mu_{2}^{\prime} \max \left\{0, \int_{0}^{2 \pi} \log \frac{1}{\left|f^{\prime}\left(s e^{i \theta}\right)\right|_{\omega_{A \times S}}} \frac{d \theta}{2 \pi}\right\}+\mu_{3}^{\prime}
\end{aligned}
$$

for $r \in(s, 1)$ outside some exceptional set $E_{2}$ with $\left|E_{2}\right|<\delta / 2$. Thus our estimate is valid for $k$.

\section{Nevanlinna theory and blowing-ups.}

We use the notion of Weil functions (cf., e.g., [35, Definition 2.2.1]). Let $V$ be a smooth projective variety and let $Z \subset V$ be a closed subscheme. A Weil function $\lambda_{Z}$ for $Z$ is a continuous function $\lambda_{Z}: V-\operatorname{supp} Z \rightarrow \mathbb{R}$ which satisfies the following condition. For each $x \in V$, there are a Zariski open neighborhood $U \subset V$ of $x$, holomorphic functions $g_{1}, \ldots, g_{l} \in \Gamma\left(U, \mathcal{O}_{V}\right)$ which defines $Z \cap U$, and a continuous function $\alpha: U \rightarrow \mathbb{R}$ on $U$ such that

$$
\left|\lambda_{Z}(y)+\log \max _{1 \leq i \leq l}\left\{\left|g_{i}(y)\right|\right\}\right| \leq \alpha(y)
$$

for all $y \in U-\operatorname{supp}(Z \cap U)$. We summarize the needed properties of Weil functions (cf. $[35$, Section 2.2]):

- If $\lambda_{Z}$ and $\lambda_{Z}^{\prime}$ are Weil functions for $Z$, then there exists a positive constant $\gamma$ such that $\left|\lambda_{Z}(x)-\lambda_{Z}^{\prime}(x)\right| \leq \gamma$ for all $x \in V-\operatorname{supp} Z$.

- Let $D$ be an effective Cartier divisor on $V$. Let $L$ be a line bundle on $V$ associated to $D$, and let $h$ be a smooth Hermitian metric on $L$. Let $\sigma$ be a section of $L$ associated to $D$ such that $h(\sigma(x), \sigma(x)) \leq 1$ for all $x \in V$. Then $\lambda_{D}(x)=-\log \sqrt{h(\sigma(x), \sigma(x))}$, where $x \in V-\operatorname{supp} D$, is a Weil function for $D$ with $\lambda_{D} \geq 0$.

- Suppose $Z=D_{1} \cap \cdots \cap D_{l}$ as closed subschemes of $V$, where $D_{1}, \ldots, D_{l}$ are effective Cartier divisors on $V$. Then $\min \left\{\lambda_{D_{1}}, \ldots, \lambda_{D_{l}}\right\}$ is a Weil function for $Z$.

- Suppose that $p: \tilde{V} \rightarrow V$ is a morphism from another smooth projective variety $\tilde{V}$. Then $\lambda_{Z} \circ p$ is a Weil function for the pull-back $p^{*} Z \subset \tilde{V}$.

By the second and the third property, we may chose a Weil function $\lambda_{Z}$ such that $\lambda_{Z} \geq 0$.

We introduce one terminology from [20, p.242]. Let $\gamma>0$ and let $W \subset \mathbb{C}$ be an open set. An assertion concerning points $w \in W$ will be said to hold for $\gamma$-almost all $w \in W$ if it holds for all $w \in W$ possibly except for $w$ contained in at most countably many closed discs such that the sum of the radii is less than $\gamma$.

LEMMA 6.1. Let $V$ be a smooth projective variety and let $Z \subset V$ be a closed subscheme such that the blow-up $\mathrm{Bl}_{Z} V$ is smooth. Let $\omega_{V}$ and $\omega_{\mathrm{Bl}_{Z} V}$ be smooth positive $(1,1)$-forms on $V$ and $\mathrm{Bl}_{Z} V$, respectively. Let $\lambda_{Z}: V-\operatorname{supp} Z \rightarrow \mathbb{R}_{\geq 0}$ be a Weil function 
for $Z$. Let $Z_{1}, \ldots, Z_{l}$ be the connected components of $Z$. For $k=1, \ldots$, l, let $U_{k} \subset V$ be an open neighborhood of supp $Z_{k}$. Let $0<\gamma<1, \delta>0$ and $0<s<1$. Then there exist positive constants $\beta_{1}, \beta_{2}$ with the following property: Let $f \in \operatorname{Hol}(\mathbb{D}, V)$ be a holomorphic map with $f(\mathbb{D}) \not \subset \operatorname{supp} Z$. Set

$$
\sigma_{f, k}=\sup \left\{t \in(0,1) \mid f(w) \in U_{k} \text { for } \gamma \text {-almost all } w \in \mathbb{D}(t)\right\}
$$

and $\sigma_{f}=\max \left\{s, \sigma_{f, 1}, \ldots, \sigma_{f, l}\right\}$. Then the estimate

$$
\int_{0}^{2 \pi} \lambda_{Z}\left(f\left(r e^{i \theta}\right)\right) \frac{d \theta}{2 \pi}+\int_{s}^{r} d t \int_{\mathbb{D}(t)} \hat{f}^{*} \omega_{\mathrm{Bl}_{Z} V} \leq \beta_{1} \int_{s}^{r} d t \int_{\mathbb{D}(t)} f^{*} \omega_{V}+\beta_{2}
$$

holds for $r \in(s, 1) \cap\left(\sigma_{f}+\delta,+\infty\right)$, where $\hat{f}: \mathbb{D} \rightarrow \mathrm{Bl}_{Z} V$ is the lifting of $f$.

We note that $\gamma \leq \sigma_{f, k} \leq 1$ for $k=1, \ldots, l$.

REMARK 6.2. By the assumption $\lambda_{Z} \geq 0$, the estimate of Lemma 6.1 yields the following estimate

$$
\int_{s}^{r} d t \int_{\mathbb{D}(t)} \hat{f}^{*} \omega_{\mathrm{Bl}_{Z} V} \leq \beta_{1} \int_{s}^{r} d t \int_{\mathbb{D}(t)} f^{*} \omega_{V}+\beta_{2}
$$

By contrast to this estimate, there is no pointwise estimate for $\left|\hat{f}^{\prime}\right|_{\omega_{\mathrm{Bl}_{Z} V}}$ from above using linear function of $\left|f^{\prime}\right|_{\omega_{V}}$ (cf. Example 2.2).

We start the proof of Lemma 6.1 from the following two preliminary estimates.

Lemma 6.3. Let $V, Z, \omega_{V}, \omega_{\mathrm{Bl}_{Z} V}$ and $\lambda_{Z}$ be the same as in Lemma 6.1. Then there exist positive constants $\alpha_{1}, \alpha_{2}$ and $\alpha_{3}$ with the following property: Let $0<s<s^{\prime}<1$. For every $f \in \operatorname{Hol}(\mathbb{D}, V)$ with $f(\mathbb{D}) \not \subset \operatorname{supp} Z$, we have

$$
\begin{aligned}
& \int_{0}^{2 \pi} \lambda_{Z}\left(f\left(r e^{i \theta}\right)\right) \frac{d \theta}{2 \pi}+\int_{s}^{r} d t \int_{\mathbb{D}(t)} \hat{f}^{*} \omega_{\mathrm{Bl}_{Z} V} \\
& \quad \leq \frac{\alpha_{1}}{s} \int_{s}^{r} d t \int_{\mathbb{D}(t)} f^{*} \omega_{V}+\alpha_{2} \int_{0}^{2 \pi} \lambda_{Z}\left(f\left(s^{\prime \prime} e^{i \theta}\right)\right) \frac{d \theta}{2 \pi}+\alpha_{3}
\end{aligned}
$$

for all $r \in\left(s^{\prime}, 1\right)$, where $s^{\prime \prime}=\left(s+s^{\prime}\right) / 2$ and $\hat{f}: \mathbb{D} \rightarrow \mathrm{Bl}_{Z} V$ is the lift of $f$.

To estimate the second term in the right hand side of (6.2), we need another

Lemma 6.4. Let $V, Z, \omega_{V}, \omega_{\mathrm{Bl}_{Z} V}$ and $\lambda_{Z}$ be the same as in Lemma 6.1. Then there exist positive constants $\alpha_{4}>0, \alpha_{5}>0$ and $\alpha_{6}>0$ with the following property: Let $0<s<s^{\prime \prime}<1$. For every $f \in \operatorname{Hol}(\mathbb{D}, V)$ with $f(\mathbb{D}) \not \subset \operatorname{supp} Z$ and every biholomorphic mapping $Q: \mathbb{D} \rightarrow \mathbb{D}\left(s^{\prime \prime}\right)$ with $Q(0) \in \mathbb{D}(s)$ and $f \circ Q(0) \notin \operatorname{supp} Z$, we have

$$
\int_{0}^{2 \pi} \lambda_{Z}\left(f\left(s^{\prime \prime} e^{i \theta}\right)\right) \frac{d \theta}{2 \pi} \leq \frac{\alpha_{4}}{s^{\prime \prime}-s} \int_{0}^{1} \frac{d t}{t} \int_{\mathbb{D}(t)}(f \circ Q)^{*} \omega_{V}+\frac{\alpha_{5}}{s^{\prime \prime}-s} \lambda_{Z}(f \circ Q(0))+\frac{\alpha_{6}}{s^{\prime \prime}-s} .
$$


We first prove Lemmas 6.3 and 6.4 .

Proof of Lemma 6.3. For $r \in\left(s^{\prime}, 1\right)$, we have

$$
\begin{aligned}
\int_{s}^{r} d t \int_{\mathbb{D}(t)} \hat{f}^{*} \omega_{\mathrm{Bl}_{Z} V} & =\int_{s}^{s^{\prime \prime}} d t \int_{\mathbb{D}(t)} \hat{f}^{*} \omega_{\mathrm{Bl}_{Z} V}+\int_{s^{\prime \prime}}^{r} d t \int_{\mathbb{D}(t)} \hat{f}^{*} \omega_{\mathrm{Bl}_{Z} V} \\
& \leq \frac{s^{\prime \prime}-s}{r-s^{\prime \prime}} \int_{s^{\prime \prime}}^{r} d t \int_{\mathbb{D}(t)} \hat{f}^{*} \omega_{\mathrm{Bl}_{Z} V}+\int_{s^{\prime \prime}}^{r} d t \int_{\mathbb{D}(t)} \hat{f}^{*} \omega_{\mathrm{Bl}_{Z} V} \\
& =\frac{r-s}{r-s^{\prime \prime}} \int_{s^{\prime \prime}}^{r} d t \int_{\mathbb{D}(t)} \hat{f}^{*} \omega_{\mathrm{Bl}_{Z} V} \\
& \leq 2 \int_{s^{\prime \prime}}^{r} \frac{d t}{t} \int_{\mathbb{D}(t)} \hat{f}^{*} \omega_{\mathrm{Bl}_{Z} V}
\end{aligned}
$$

Hence, for $r \in\left(s^{\prime}, 1\right)$, we have

$$
\int_{s}^{r} d t \int_{\mathbb{D}(t)} \hat{f}^{*} \omega_{\mathrm{Bl}_{Z} V} \leq 2 \int_{s^{\prime \prime}}^{r} \frac{d t}{t} \int_{\mathbb{D}(t)} \hat{f}^{*} \omega_{\mathrm{Bl}_{Z} V}
$$

Let $p: \mathrm{Bl}_{Z} V \rightarrow V$ be the projection and let $p^{*} Z \subset \mathrm{Bl}_{Z} V$ be the induced closed subscheme. Then $p^{*} Z$ is a Cartier divisor on $\mathrm{Bl}_{Z} V$. We denote by $L$ the associated line bundle. Let $M$ be an ample line bundle on $V$. Then there exists a positive integer $l$ such that $p^{*} M^{\otimes l} \otimes L^{-1}$ is ample on $\mathrm{Bl}_{Z} V$. Hence there exist smooth Hermitian metrics $h_{L}$ on $L$ and $h_{M}$ on $M$ such that

$$
l p^{*} c_{1}\left(M, h_{M}\right)-c_{1}\left(L, h_{L}\right)
$$

is a positive $(1,1)$-form on $\mathrm{Bl}_{Z} V$. Here $c_{1}(\cdot)$ are associated curvature forms. Thus there exist positive constants $\gamma_{1}>1$ and $\gamma_{2}>1$, which depend on $\omega_{\mathrm{Bl}_{Z} V}, \omega_{V},\left(L, h_{L}\right),\left(M, h_{M}\right)$, such that

$$
\omega_{\mathrm{Bl}_{Z} V} \leq \gamma_{1} p^{*} \omega_{V}-\gamma_{2} c_{1}\left(L, h_{L}\right)
$$

We have

$$
\int_{s^{\prime \prime}}^{r} \frac{d t}{t} \int_{\mathbb{D}(t)} \hat{f}^{*} \omega_{\mathrm{Bl}_{Z} V} \leq \gamma_{1} \int_{s^{\prime \prime}}^{r} \frac{d t}{t} \int_{\mathbb{D}(t)} f^{*} \omega_{V}-\gamma_{2} \int_{s^{\prime \prime}}^{r} \frac{d t}{t} \int_{\mathbb{D}(t)} \hat{f}^{*} c_{1}\left(L, h_{L}\right)
$$

for $r \in\left(s^{\prime \prime}, 1\right)$. Let $\sigma$ be the section of $L$ associated to $p^{*} Z$. We may assume that $h_{L}(\sigma(x), \sigma(x)) \leq 1$ for all $x \in \mathrm{Bl}_{Z} V$. We set $\lambda_{p^{*} Z}(x)=-\log \sqrt{h_{L}(\sigma(x), \sigma(x))}$, where $x \in \mathrm{Bl}_{Z} V-\operatorname{supp} p^{*} Z$. Then by the first main theorem (cf. [28, Theorem 2.3.31]), we have

$$
\int_{s^{\prime \prime}}^{r} \frac{d t}{t} \int_{\mathbb{D}(t)} \hat{f}^{*} c_{1}\left(L, h_{L}\right) \geq \int_{0}^{2 \pi} \lambda_{p^{*} Z}\left(\hat{f}\left(r e^{i \theta}\right)\right) \frac{d \theta}{2 \pi}-\int_{0}^{2 \pi} \lambda_{p^{*} Z}\left(\hat{f}\left(s^{\prime \prime} e^{i \theta}\right)\right) \frac{d \theta}{2 \pi}
$$

for $r \in\left(s^{\prime \prime}, 1\right)$. Since $\lambda_{Z} \circ p$ is a Weil function for $p^{*} Z$, there exists a positive constant $\gamma_{3}$, which depends on $\left(L, h_{L}\right), \sigma, \lambda_{Z}$, such that 


$$
\lambda_{Z}(p(x))-\gamma_{3} \leq \lambda_{p^{*} Z}(x) \leq \lambda_{Z}(p(x))+\gamma_{3}
$$

for all $x \in \mathrm{Bl}_{Z} V-\operatorname{supp} p^{*} Z$. Hence we have

$$
\begin{aligned}
& \int_{0}^{2 \pi} \lambda_{p^{*} Z}\left(\hat{f}\left(s^{\prime \prime} e^{i \theta}\right)\right) \frac{d \theta}{2 \pi}-\int_{0}^{2 \pi} \lambda_{p^{*} Z}\left(\hat{f}\left(r e^{i \theta}\right)\right) \frac{d \theta}{2 \pi} \\
& \quad \leq \int_{0}^{2 \pi} \lambda_{Z}\left(f\left(s^{\prime \prime} e^{i \theta}\right)\right) \frac{d \theta}{2 \pi}-\int_{0}^{2 \pi} \lambda_{Z}\left(f\left(r e^{i \theta}\right)\right) \frac{d \theta}{2 \pi}+2 \gamma_{3} .
\end{aligned}
$$

Hence by (6.5)-(6.7), we obtain

$$
\begin{aligned}
& \gamma_{2} \int_{0}^{2 \pi} \lambda_{Z}\left(f\left(r e^{i \theta}\right)\right) \frac{d \theta}{2 \pi}+\int_{s^{\prime \prime}}^{r} \frac{d t}{t} \int_{\mathbb{D}(t)} \hat{f}^{*} \omega_{\mathrm{Bl}_{Z} V} \\
& \quad \leq \gamma_{1} \int_{s^{\prime \prime}}^{r} \frac{d t}{t} \int_{\mathbb{D}(t)} f^{*} \omega_{V}+\gamma_{2} \int_{0}^{2 \pi} \lambda_{Z}\left(f\left(s^{\prime \prime} e^{i \theta}\right)\right) \frac{d \theta}{2 \pi}+2 \gamma_{2} \gamma_{3} .
\end{aligned}
$$

By $\gamma_{2}>1$ and

$$
\int_{s^{\prime \prime}}^{r} \frac{d t}{t} \int_{\mathbb{D}(t)} f^{*} \omega_{V} \leq \frac{1}{s} \int_{s}^{r} d t \int_{\mathbb{D}(t)} f^{*} \omega_{V}
$$

we get

$$
\begin{aligned}
& \int_{0}^{2 \pi} \lambda_{Z}\left(f\left(r e^{i \theta}\right)\right) \frac{d \theta}{2 \pi}+\int_{s^{\prime \prime}}^{r} \frac{d t}{t} \int_{\mathbb{D}(t)} \hat{f}^{*} \omega_{\mathrm{Bl}_{Z} V} \\
& \quad \leq \frac{\gamma_{1}}{s} \int_{s}^{r} d t \int_{\mathbb{D}(t)} f^{*} \omega_{V}+\gamma_{2} \int_{0}^{2 \pi} \lambda_{Z}\left(f\left(s^{\prime \prime} e^{i \theta}\right)\right) \frac{d \theta}{2 \pi}+2 \gamma_{2} \gamma_{3} .
\end{aligned}
$$

Combining this estimate with (6.4), we obtain (6.2). Here we set $\alpha_{1}=2 \gamma_{1}, \alpha_{2}=2 \gamma_{2}$, and $\alpha_{3}=4 \gamma_{2} \gamma_{3}$.

Proof of Lemma 6.4. Let $D_{1}, \cdots, D_{\nu}$ be effective ample divisors such that $\lambda_{Z}=\min \left\{\lambda_{D_{i}}\right\}$. We have, for $i=1, \ldots, \nu$ with $f(\mathbb{D}) \not \subset \operatorname{supp} D_{i}$,

$$
\begin{aligned}
\int_{0}^{2 \pi} \lambda_{Z}\left(f\left(s^{\prime \prime} e^{i \theta}\right)\right) \frac{d \theta}{2 \pi} & \leq \frac{s^{\prime \prime}+s}{s^{\prime \prime}-s} \int_{0}^{2 \pi} \lambda_{Z}\left(f \circ Q\left(e^{i \theta}\right)\right) \frac{d \theta}{2 \pi} \\
& \leq \frac{2}{s^{\prime \prime}-s} \int_{0}^{2 \pi} \lambda_{D_{i}}\left(f \circ Q\left(e^{i \theta}\right)\right) \frac{d \theta}{2 \pi} .
\end{aligned}
$$

By the first main theorem, there exist positive constants $\gamma_{4}$ and $\gamma_{5}$ which depend on $\omega_{V}$ and $\left\{\lambda_{D_{i}}\right\}$ such that

$$
\int_{0}^{2 \pi} \lambda_{D_{i}}\left(f \circ Q\left(e^{i \theta}\right)\right) \frac{d \theta}{2 \pi} \leq \gamma_{4} \int_{0}^{1} \frac{d t}{t} \int_{\mathbb{D}(t)}(f \circ Q)^{*} \omega_{V}+\lambda_{D_{i}}(f \circ Q(0))+\gamma_{5}
$$

for $i=1, \ldots, \nu$ with $f(\mathbb{D}) \not \subset \operatorname{supp} D_{i}$. Hence we get 


$$
\int_{0}^{2 \pi} \lambda_{Z}\left(f\left(s^{\prime \prime} e^{i \theta}\right)\right) \frac{d \theta}{2 \pi} \leq \frac{2 \gamma_{4}}{s^{\prime \prime}-s} \int_{0}^{1} \frac{d t}{t} \int_{\mathbb{D}(t)}(f \circ Q)^{*} \omega_{V}+\frac{2}{s^{\prime \prime}-s} \lambda_{D_{i}}(f \circ Q(0))+\frac{2 \gamma_{5}}{s^{\prime \prime}-s}
$$

for $i=1, \ldots, \nu$ with $f(\mathbb{D}) \not \subset \operatorname{supp} D_{i}$. We take $i$ such that $\lambda_{Z}(f \circ Q(0))=\lambda_{D_{i}}(f \circ Q(0))$ to conclude the proof of (6.3). Here we set $\alpha_{4}=2 \gamma_{4}, \alpha_{5}=2$, and $\alpha_{6}=2 \gamma_{5}$.

Before going to prove Lemma 6.1, we quote the Bloch-Cartan estimate (cf. [13, Lemma 6.17]): If $\mu$ is a mass distribution on $\mathbb{C}$ with finite total mass $M$ and $\gamma$ is a constant with $0<\gamma<1$, then we have

$$
\int_{\mathbb{C}} \log \frac{1}{|z-w|} d \mu_{z} \leq \tau_{\gamma} M
$$

for $\gamma$-almost all $w \in \mathbb{C}$, where $\tau_{\gamma}>0$ is a positive constant which depends on $\gamma$. For instance, we may take as $\tau_{\gamma}=\log (6 / \gamma)$. This estimate is due to Bloch [1] and Cartan [5]. See also [20, VIII, Section 3].

Proof of Lemma 6.1. If $\sigma_{f}+\delta \geq 1$, then our claim is trivial. Hence in the following, we assume $\sigma_{f}<1-\delta$. Set $s_{f}=\sigma_{f}+\delta / 2, s_{f}^{\prime}=\sigma_{f}+\delta$ and $s_{f}^{\prime \prime}=\left(s_{f}+s_{f}^{\prime}\right) / 2$. We remark that $s_{f}>s$ and that $s_{f}^{\prime \prime}-s_{f}=\delta / 4$ does not depend on the choice of $f$. For each $k=1, \ldots, l$, let $\lambda_{Z_{k}}: V-\operatorname{supp} Z_{k} \rightarrow \mathbb{R}_{\geq 0}$ be a Weil function for $Z_{k}$. Then $\lambda_{Z_{1}}+\cdots+\lambda_{Z_{l}}$ is a Weil function for $Z$. By the estimate (6.2), we obtain the following: There exist positive constants $\alpha_{1}, \alpha_{2}$ and $\alpha_{3}$ such that, for each $f \in \operatorname{Hol}(\mathbb{D}, V)$ with $f(\mathbb{D}) \not \subset \operatorname{supp} Z$, we have

$$
\begin{aligned}
& \int_{0}^{2 \pi} \lambda_{Z}\left(f\left(r e^{i \theta}\right)\right) \frac{d \theta}{2 \pi}+\int_{s_{f}}^{r} d t \int_{\mathbb{D}(t)} \hat{f}^{*} \omega_{\mathrm{Bl}_{Z} V} \\
& \quad \leq \frac{\alpha_{1}}{s_{f}} \int_{s_{f}}^{r} d t \int_{\mathbb{D}(t)} f^{*} \omega_{V}+\alpha_{2} \sum_{k=1}^{l} \int_{0}^{2 \pi} \lambda_{Z_{k}}\left(f\left(s_{f}^{\prime \prime} e^{i \theta}\right)\right) \frac{d \theta}{2 \pi}+\alpha_{3}
\end{aligned}
$$

for $r \in\left(s_{f}^{\prime}, 1\right)$. We have

$$
\begin{aligned}
\int_{s}^{r} d t \int_{\mathbb{D}(t)} \hat{f}^{*} \omega_{\mathrm{Bl}_{Z} V} & =\int_{s}^{s_{f}} d t \int_{\mathbb{D}(t)} \hat{f}^{*} \omega_{\mathrm{Bl}_{Z} V}+\int_{s_{f}}^{r} d t \int_{\mathbb{D}(t)} \hat{f}^{*} \omega_{\mathrm{Bl}_{Z} V} \\
& \leq \frac{s_{f}-s}{r-s_{f}} \int_{s_{f}}^{r} d t \int_{\mathbb{D}(t)} \hat{f}^{*} \omega_{\mathrm{Bl}_{Z} V}+\int_{s_{f}}^{r} d t \int_{\mathbb{D}(t)} \hat{f}^{*} \omega_{\mathrm{Bl}_{Z} V} \\
& =\frac{r-s}{r-s_{f}} \int_{s_{f}}^{r} d t \int_{\mathbb{D}(t)} \hat{f}^{*} \omega_{\mathrm{Bl}_{Z} V} \\
& \leq \frac{2(1-s)}{\delta} \int_{s_{f}}^{r} d t \int_{\mathbb{D}(t)} \hat{f}^{*} \omega_{\mathrm{Bl}_{Z} V}
\end{aligned}
$$

for $r \in\left(s_{f}^{\prime}, 1\right)$. Hence by $2(1-s) / \delta>1$, we have

$$
\int_{0}^{2 \pi} \lambda_{Z}\left(f\left(r e^{i \theta}\right)\right) \frac{d \theta}{2 \pi}+\int_{s}^{r} d t \int_{\mathbb{D}(t)} \hat{f}^{*} \omega_{\mathrm{Bl}_{Z} V} \leq \frac{2 \alpha_{1}(1-s)}{s \delta} \int_{s}^{r} d t \int_{\mathbb{D}(t)} f^{*} \omega_{V}
$$




$$
+\frac{2 \alpha_{2}(1-s)}{\delta} \sum_{k=1}^{l} \int_{0}^{2 \pi} \lambda_{Z_{k}}\left(f\left(s_{f}^{\prime \prime} e^{i \theta}\right)\right) \frac{d \theta}{2 \pi}+\frac{2 \alpha_{3}(1-s)}{\delta}
$$

for $r \in\left(s_{f}^{\prime}, 1\right)$.

For each $Z_{k}$, we apply the estimate (6.3) to get the following: There exist positive constants $\alpha_{4, k}>0, \alpha_{5, k}>0$ and $\alpha_{6, k}>0$ such that, for each $f \in \operatorname{Hol}(\mathbb{D}, V)$ with $f(\mathbb{D}) \not \subset$ $\operatorname{supp} Z_{k}$ and for each biholomorphic mapping $Q_{k}: \mathbb{D} \rightarrow \mathbb{D}\left(s_{f}^{\prime \prime}\right)$ with $Q_{k}(0) \in \mathbb{D}\left(s_{f}\right)$ and $f \circ Q_{k}(0) \notin \operatorname{supp} Z_{k}$, we have

$$
\int_{0}^{2 \pi} \lambda_{Z_{k}}\left(f\left(s_{f}^{\prime \prime} e^{i \theta}\right)\right) \frac{d \theta}{2 \pi} \leq \frac{4 \alpha_{4, k}}{\delta} \int_{0}^{1} \frac{d t}{t} \int_{\mathbb{D}(t)}\left(f \circ Q_{k}\right)^{*} \omega_{V}+\frac{4 \alpha_{5, k}}{\delta} \lambda_{Z_{k}}\left(f \circ Q_{k}(0)\right)+\frac{4 \alpha_{6, k}}{\delta} .
$$

Set $\alpha_{4}=\max _{1 \leq k \leq l}\left\{4 \alpha_{4, k} / \delta\right\}, \alpha_{5}=\max _{1 \leq k \leq l}\left\{4 \alpha_{5, k} / \delta\right\}$ and $\alpha_{6}=\sum_{k=1}^{l} 4 \alpha_{6, k} / \delta$. Then we get

$$
\sum_{k=1}^{l} \int_{0}^{2 \pi} \lambda_{Z_{k}}\left(f\left(s_{f}^{\prime \prime} e^{i \theta}\right)\right) \frac{d \theta}{2 \pi} \leq \alpha_{4} \sum_{k=1}^{l} \int_{0}^{1} \frac{d t}{t} \int_{\mathbb{D}(t)}\left(f \circ Q_{k}\right)^{*} \omega_{V}+\alpha_{5} \sum_{k=1}^{l} \lambda_{Z_{k}}\left(f \circ Q_{k}(0)\right)+\alpha_{6} .
$$

Now we chose $Q_{k}$. Let $\mu$ be a muss distribution on $\mathbb{C}$ defined by $\mu=\mathbb{I}_{\mathbb{D}\left(s_{f}^{\prime \prime}\right)} f^{*} \omega_{V}$. Then $\mu$ has finite total mass $\int_{\mathbb{D}\left(s_{f}^{\prime \prime}\right)} f^{*} \omega_{V}$. We apply the Bloch-Cartan estimate (6.8) to conclude that, for each $1 \leq k \leq l$, there exists $w_{k} \in \mathbb{D}\left(s_{f}\right)$ such that $f\left(w_{k}\right) \notin U_{k}$ and

$$
\int_{\mathbb{C}} \log \frac{1}{\left|z-w_{k}\right|} d \mu_{z} \leq \tau_{\gamma} \int_{\mathbb{D}\left(s_{f}^{\prime \prime}\right)} f^{*} \omega_{V}
$$

Indeed otherwise, we have $f(w) \in U_{k}$ for $\gamma$-almost all $w \in \mathbb{D}\left(s_{f}\right)$, which contradicts to the choice of $s_{f}$. Let $Q_{k}: \mathbb{D} \rightarrow \mathbb{D}\left(s_{f}^{\prime \prime}\right)$ be a biholomorphic mapping such that $Q_{k}(0)=w_{k}$. Then we have

$$
\begin{aligned}
\int_{0}^{1} \frac{d t}{t} \int_{\mathbb{D}(t)}\left(f \circ Q_{k}\right)^{*} \omega_{V} & =\int_{\mathbb{C}} \log \frac{1}{|\xi|} d\left(Q_{k}^{*} \mu\right)_{\xi}=\int_{\mathbb{C}} \log \frac{1}{\left|Q_{k}^{-1}(z)\right|} d \mu_{z} \\
& =\int_{\mathbb{C}} \log \frac{\left|s_{f}^{\prime \prime}-\left(\overline{w_{k}} / s_{f}^{\prime \prime}\right) z\right|}{\left|z-w_{k}\right|} d \mu_{z} \leq\left(\tau_{\gamma}+\log 2\right) \int_{\mathbb{D}\left(s_{f}^{\prime \prime}\right)} f^{*} \omega_{V}
\end{aligned}
$$

Hence, for $r \in\left(s_{f}^{\prime}, 1\right)$, we have

$$
\int_{0}^{1} \frac{d t}{t} \int_{\mathbb{D}(t)}\left(f \circ Q_{k}\right)^{*} \omega_{V} \leq \frac{4\left(\tau_{\gamma}+\log 2\right)}{\delta} \int_{s}^{r} d t \int_{\mathbb{D}(t)} f^{*} \omega_{V}
$$

We set $\eta_{k}=\sup _{z \in V-U_{k}} \lambda_{Z_{k}}(z)$ and $\eta=\max _{1 \leq k \leq l} \eta_{k}$. Then we have

$$
\lambda_{Z_{k}}\left(f \circ Q_{k}(0)\right) \leq \eta
$$

Combining (6.9)-(6.12), we get our lemma. 


\section{Application of a covering lemma.}

Lemma 7.1. Let $A$ be an abelian variety and let $\omega_{A}$ be a positive invariant $(1,1)$ form on $A$. Let $0<s<\sigma<1$ and $\delta>0$. Let $F \subset \mathbb{D}(\sigma)$ be a relatively closed subset such that for all $\sigma^{\prime} \in(0, \sigma)$, the set $F \cap \mathbb{D}\left(\sigma^{\prime}\right)$ is covered by at most countably many closed discs whose sum of radii is less than $\delta / 800$. Then for every $f \in \operatorname{Hol}(\mathbb{D}, A)$, the estimate

$$
\int_{s}^{r} d t \int_{\mathbb{D}(t)} f^{*} \omega_{A} \leq 4 \int_{s}^{r} d t \int_{\mathbb{D}(t) \backslash F} f^{*} \omega_{A}
$$

holds for $r \in(s, \sigma)$ outside some exceptional set whose linear measure is less than $\delta$.

Proof. $\quad$ Let $P \subset(0, \sigma)$ be defined by

$$
P=\{r \in(0, \sigma) ;\{|z|=r\} \cap F \neq \emptyset\} .
$$

Then $|P| \leq \delta / 400$. For $0 \leq t<1$, we set

$$
\varphi(t)=\int_{0}^{2 \pi}\left|f^{\prime}\left(t e^{i \theta}\right)\right|_{\omega_{A}}^{2} d \theta
$$

Then since $\left|f^{\prime}(z)\right|_{\omega_{A}}^{2}$ is subharmonic (cf. (2.1)), $\varphi(t)$ is a non-negative, increasing function. We apply Lemma 7.2 below to get

$$
\int_{\mathbb{D}(r)} f^{*} \omega_{A}=\int_{0}^{r} \varphi(t) t d t \leq 2 \int_{(0, r) \backslash P} \varphi(t) t d t \leq 2 \int_{\mathbb{D}(r) \backslash F} f^{*} \omega_{A}
$$

for $r \in(0, \sigma)$ outside some exceptional set $E \subset(0, \sigma)$ whose linear measure is less than $\delta / 20$. Again we apply Lemma 7.2 to get

$\int_{s}^{r} d t \int_{\mathbb{D}(t)} f^{*} \omega_{A} \leq 2 \int_{[s, r] \backslash E} d t \int_{\mathbb{D}(t)} f^{*} \omega_{A} \leq 4 \int_{[s, r] \backslash E} d t \int_{\mathbb{D}(t) \backslash F} f^{*} \omega_{A} \leq 4 \int_{s}^{r} d t \int_{\mathbb{D}(t) \backslash F} f^{*} \omega_{A}$ for $r \in(s, \sigma)$ outside some exceptional set whose linear measure is less than $\delta$.

Lemma 7.2. Let $\varphi:[0,1) \rightarrow \mathbb{R}_{\geq 0}$ be a non-negative, increasing function. Let $0 \leq u<u^{\prime}<1$ and $\gamma>0$. Let $P \subset\left(0, u^{\prime}\right)$ be a subset such that $|P| \leq \gamma$. Then we have

$$
\int_{[u, r]} \varphi(x) d x \leq 2 \int_{[u, r]-P} \varphi(x) d x
$$

for $r \in\left(u, u^{\prime}\right)$ outside some exceptional set whose linear measure is less than $20 \gamma$.

Proof. We set

$$
E=\left\{x \in\left(u, u^{\prime}\right) ; \exists t>0 \text { s.t. }|[x-t, x] \cap P|>\frac{t}{2}\right\} .
$$

We shall show that $|E|<20 \gamma$. For $x \in E$, we take $t_{x}>0$ such that 


$$
\left|\left[x-t_{x}, x\right] \cap P\right|>t_{x} / 2 .
$$

We set $I_{x}=\left[x-t_{x}, x+t_{x}\right]$. Then

$$
E \subset \bigcup_{x \in E} I_{x}
$$

By the Vitali covering theorem [8, p.27], there exists a countable set $Q \subset E$ such that, letting $I_{x}^{\prime}=\left[x-5 t_{x}, x+5 t_{x}\right]$, the family $\left\{I_{x}\right\}_{x \in Q}$ are disjoint and

$$
E \subset \bigcup_{x \in Q} I_{x}^{\prime}
$$

By (7.1), we have

$$
\left|I_{x} \cap P\right|>\left|I_{x}^{\prime}\right| / 20 .
$$

Hence we have

$$
|E| \leq \sum_{x \in Q}\left|I_{x}^{\prime}\right|<20 \sum_{x \in Q}\left|I_{x} \cap P\right| \leq 20|P| \leq 20 \gamma .
$$

Now let $r \in\left(u, u^{\prime}\right)-E$. Then for all $a \leq r$, we have

$$
|[a, r]-P| \geq \frac{1}{2}|[a, r]| .
$$

Hence, for $r \in\left(u, u^{\prime}\right)-E$, we have

$$
\begin{aligned}
\int_{[u, r]-P} \varphi(x) d x & =\int_{0}^{\varphi(r)}\left|\left[\max \left\{u, \varphi^{-1}(y)\right\}, r\right]-P\right| d y \\
& \geq \frac{1}{2} \int_{0}^{\varphi(r)}\left|\left[\max \left\{u, \varphi^{-1}(y)\right\}, r\right]\right| d y=\frac{1}{2} \int_{[u, r]} \varphi(x) d x
\end{aligned}
$$

where $\varphi^{-1}(y)=\sup \{x \in[0,1) ; \varphi(x)<y\}$.

\section{Proof of Proposition 2.3.}

We first recall the principle of transfinite induction. Let $I$ be a totally ordered set which is well-ordered, i.e., every non-empty subset of $I$ has a minimum element. Let $\mathbf{P}(i)$ be a property parametrized by $i \in I$.

LEMma 8.1. For all $i \in I$, assume that:

$$
\text { If } \mathbf{P}(j) \text { holds for all } j<i \text {, then } \mathbf{P}(i) \text {. }
$$

Then $\mathbf{P}(i)$ holds for all $i \in I$.

Proof. We define a subset $J \subset I$ by 


$$
J=\{i \in I ; \mathbf{P}(i) \text { does not hold }\} .
$$

To prove that $J$ is an empty set, we assume contrary that $J \neq \emptyset$. Since $I$ is well-ordered, we may take the minimum element $j_{0} \in J$. For all $i<j_{0}$, we have $i \notin J$, hence $\mathbf{P}(i)$ holds. Hence $\mathbf{P}\left(j_{0}\right)$ holds by our assumption (8.1). This contradicts to $j_{0} \in J$. Hence $J=\emptyset$. Thus $\mathbf{P}(i)$ holds for all $i \in I$.

We return to the situation of Proposition 2.3. We fix a closed embedding $A \subset \mathbb{P}^{n}$, where $n>0$. Recall that $\mathcal{P}$ is the set of all numerical polynomials which appear as Hilbert polynomials of closed subschemes of the projective space $\mathbb{P}^{n}$. Then $\mathcal{P}$ is a totally ordered set by $P_{1} \leq P_{2}$ if and only if $P_{1}(m) \leq P_{2}(m)$ for all large integers $m$.

\section{LEMMA 8.2. The set $\mathcal{P}$ is well-ordered.}

Proof. Let $P_{\mathbb{P}^{n}}$ be the Hilbert polynomial of $\mathbb{P}^{n}$. Then $P_{\mathbb{P}^{n}}$ is a maximum element of $\mathcal{P}$. Let $J \subset \mathcal{P}$ be a non-empty subset. We shall show that $J$ has a minimum element. We may assume $J \backslash\left\{P_{\mathbb{P}^{n}}\right\} \neq \emptyset$, for otherwise $P_{\mathbb{P}^{n}}$ is the minimum element of $J$. Note that $\mathcal{P} \backslash\left\{P_{\mathbb{P}^{n}}\right\}$ is the set of all Hilbert polynomials of proper closed subschemes of $\mathbb{P}^{n}$. By [11, Corollary 5.7], we may write $P \in \mathcal{P} \backslash\left\{P_{\mathbb{P}^{n}}\right\}$ as

$$
P(z)=\sum_{t=0}^{n-1}\left\{\left(\begin{array}{c}
z+t \\
t+1
\end{array}\right)-\left(\begin{array}{c}
z+t-m_{t} \\
t+1
\end{array}\right)\right\}
$$

where $m_{0}, m_{1}, \ldots, m_{n-1} \in \mathbb{Z}_{\geq 0}$ and $m_{0} \geq m_{1} \geq \cdots \geq m_{n-1} \geq 0$. We associate $P$ the sequence $\left(m_{n-1}, \ldots, m_{0}\right) \in\left(\mathbb{Z}_{\geq 0}\right)^{n}$. Then our order in $\mathcal{P} \backslash\left\{P_{\mathbb{P}^{n}}\right\}$ corresponds to the dictionary order in $\left(\mathbb{Z}_{\geq 0}\right)^{n}$. Since $\left(\mathbb{Z}_{\geq 0}\right)^{n}$ with the dictionary order is well-ordered, $J \backslash\left\{P_{\mathbb{P}^{n}}\right\}$ has the minimum element $Q$. Then $Q$ is the minimum element of $J$.

REmark 8.3. The minimum element of $\mathcal{P}$ is $P_{\emptyset}=0$ which is the Hilbert polynomial of $\emptyset \subset \mathbb{P}^{n}$.

For a closed subscheme $X \subset A \times S$, we attach $P=P_{\max } \in \mathcal{P}$ and $T \subset S$ as in (3.1), under the fixed embedding $A \subset \mathbb{P}^{n}$. Here we recall $P_{\max }=\max _{s \in S}\left\{P_{X_{s}}\right\}$.

Now we prove Proposition 2.3. We prove the proposition by the transfinite induction on $P \in \mathcal{P}$ attached to $X \rightarrow S$. So we assume that the proposition is true when the attached polynomial is less than $P$.

We first remark that if $X$ is empty or $\operatorname{dim} X=0$, then our proposition is trivially valid with $\Lambda=\{\{0\}\}$. Thus in the following, we assume $\operatorname{dim} X>0$. Then $X_{k} \subset A \times S_{[k]}$ is non-empty for all $k \geq 0$, because there exists a non-constant holomorphic map $\mathbb{D} \rightarrow X$.

We first find the non-empty finite subset $\Lambda \subset \Sigma(A)$ in the Proposition 2.3. We take $\nu>0$ such that Lemma 4.2 holds. Let $T_{[\nu]} \subset S_{[\nu]}$ be defined as (3.1) by $p_{\nu}: X_{\nu} \rightarrow S_{[\nu]}$. Let $T_{1}, \ldots, T_{l}$ be the connected components of $T_{[\nu]}$. We have

$$
l \geq 1 \text {. }
$$


For each $T_{k}$, where $k=1, \ldots, l$, we take $B_{k} \in \Sigma(A)$ as in Lemma 4.4. We define $\Lambda \subset \Sigma(A)$ in the following two cases. First, if $T_{[\nu]}=p_{\nu}\left(X_{\nu}\right)$, then we set $\Lambda=\left\{B_{1}, \ldots, B_{l}\right\}$. Next we consider the case $T_{[\nu]} \neq p_{\nu}\left(X_{\nu}\right)$. Let $\mathcal{T} \subset S_{[\nu]}$ be a closed subscheme such that $\operatorname{supp} \mathcal{T}=T_{[\nu]}$ as in Lemma 3.1. Set $\hat{S}=\mathrm{Bl}_{\mathcal{T}} S_{[\nu]}$. By Remark 3.2, we may assume that $\hat{S}$ is smooth. Let $\hat{X} \subset A \times \hat{S}$ be the scheme theoretic closure of $p_{\nu}^{-1}\left(S_{[\nu]}-T_{[\nu]}\right)$ in $\left(X_{\nu}\right) \times_{S_{[\nu]}} \hat{S}$. Let $\hat{p}: \hat{X} \rightarrow \hat{S}$ be the projection. We attach $\hat{P} \in \mathcal{P}$ to $\hat{p}: \hat{X} \rightarrow \hat{S}$. By Lemma 3.1, we have $\hat{P}<P$. Thus by the induction hypothesis, Proposition 2.3 is true for $\hat{p}: \hat{X} \rightarrow \hat{S}$. We take the non-empty finite subset $\hat{\Lambda} \subset \Sigma(A)$ which appears in Proposition 2.3 for $\hat{p}: \hat{X} \rightarrow \hat{S}$. We set $\Lambda=\hat{\Lambda} \cup\left\{B_{1}, \ldots, B_{l}\right\}$. By (8.2), we have $\Lambda \neq \emptyset$ for the both cases above.

Next we take $\omega_{A}, \omega_{S}, \omega_{A / B}, s, \varepsilon, \delta$ as in Proposition 2.3. Let $\omega_{S_{[\nu]}}$ be a smooth, positive $(1,1)$-form on $S_{[\nu]}$. By Lemma 4.4, there exist open neighborhoods $T_{1} \subset U_{1}, \ldots, T_{l} \subset U_{l}$ and positive constants $\rho_{1}, \ldots, \rho_{l}$ such that, for $k=1, \ldots, l$,

$$
\left|\left(\varpi_{B_{k}}\right)_{*}\left(\left(v_{x}\right)_{A}\right)\right|_{\omega_{A / B_{k}}}^{2} \leq \frac{\varepsilon}{8}\left|\left(v_{x}\right)_{A}\right|_{\omega_{A}}^{2}+\rho_{k}\left|\left(v_{x}\right)_{S_{[\nu]}}\right|_{\omega_{S_{[\nu]}}}^{2}
$$

for all $x \in A \times U_{k}$ and all $v_{x} \in\left(V_{\nu}\right)_{x}$. Let $\gamma=\delta / 3200$.

Now let $f \in \operatorname{Hol}(\mathbb{D}, X)$. Since the estimate of Proposition 2.3 is trivial for a constant map, we assume that $f$ is non-constant. For $k=1, \ldots, l$, we set

$$
\sigma_{k}=\sup \left\{t \in(0,1) \mid\left(f_{[\nu]}\right)_{S_{[\nu]}}(z) \in U_{k} \text { for } \gamma \text {-almost all } z \in \mathbb{D}(t)\right\} .
$$

Let $\sigma=\max \left\{s, \sigma_{1}, \ldots, \sigma_{l}\right\}$. We consider two cases.

Case 1: $r \in(s, 1) \cap(0, \sigma)$. Note that this case occurs only when $\sigma>s$. In this case, we shall not use the induction hypothesis. We take $k$ such that $\sigma_{k}=\sigma$. We set

$$
F_{k}=\left\{z \in \mathbb{D}(\sigma) ;\left(f_{[\nu]}\right)_{S_{[\nu]}}(z) \notin U_{k}\right\}
$$

Then by (8.3), we have

$$
\int_{s}^{r} d t \int_{\mathbb{D}(t) \backslash F_{k}}\left(\varpi_{B_{k}} \circ f_{A}\right)^{*} \omega_{A / B_{k}} \leq \frac{\varepsilon}{8} \int_{s}^{r} d t \int_{\mathbb{D}(t)} f_{A}^{*} \omega_{A}+\rho_{k} \int_{s}^{r} d t \int_{\mathbb{D}(t)}\left(\left(f_{[\nu]}\right)_{S_{[\nu]}}\right)^{*} \omega_{S_{[\nu]}} .
$$

Hence by Lemma 7.1, we get

$$
\int_{s}^{r} d t \int_{\mathbb{D}(t)}\left(\varpi_{B_{k}} \circ f_{A}\right)^{*} \omega_{A / B_{k}} \leq \frac{\varepsilon}{2} \int_{s}^{r} d t \int_{\mathbb{D}(t)} f_{A}^{*} \omega_{A}+4 \rho_{k} \int_{s}^{r} d t \int_{\mathbb{D}(t)}\left(\left(f_{[\nu]}\right)_{S_{[\nu]}}\right)^{*} \omega_{S_{[\nu]}}
$$

for $r \in(s, \sigma)$ outside some exceptional set of linear measure less than $\delta / 4$. Set $\rho=$ $\max \left\{\rho_{1}, \ldots, \rho_{l}\right\}$. Then we get

$\min _{B \in \Lambda}\left\{\int_{s}^{r} d t \int_{\mathbb{D}(t)}\left(\varpi_{B} \circ f_{A}\right)^{*} \omega_{A / B}\right\} \leq \frac{\varepsilon}{2} \int_{s}^{r} d t \int_{\mathbb{D}(t)} f_{A}^{*} \omega_{A}+4 \rho \int_{s}^{r} d t \int_{\mathbb{D}(t)}\left(\left(f_{[\nu]}\right)_{S_{[\nu]}}\right)^{*} \omega_{S_{[\nu]}}$

for $r \in(s, \sigma)$ outside some exceptional set of linear measure less than $\delta / 4$. 
Case 2: $r \in(s, 1) \cap(\sigma,+\infty)$. Note that this case occurs only when $T_{[\nu]} \neq p_{\nu}\left(X_{\nu}\right)$ and $\left(f_{[\nu]}\right)_{S_{[\nu]}}(\mathbb{D}) \not \subset T_{[\nu]}$. Let $\hat{f}: \mathbb{D} \rightarrow \hat{X}$ be a holomorphic map induced from $f_{[\nu]}: \mathbb{D} \rightarrow X_{\nu}$. Let $\omega_{\hat{S}}$ be a smooth, positive $(1,1)$-form on $\hat{S}$ such that $\tau^{*} \omega_{S} \leq \omega_{\hat{S}}$, where $\tau: \hat{S} \rightarrow S$ is the composition of the natural maps $\hat{S} \rightarrow S_{[\nu]} \rightarrow S$. Then

$$
\left|f^{\prime}\left(s e^{i \theta}\right)\right|_{\omega_{A \times S}} \leq\left|\hat{f}^{\prime}\left(s e^{i \theta}\right)\right|_{\omega_{A \times \hat{S}}} .
$$

By Lemma 6.1 (cf. (6.1)), we have

$$
\int_{s}^{r} d t \int_{\mathbb{D}(t)} \hat{f}_{\hat{S}}^{*} \omega_{\hat{S}} \leq \beta_{1} \int_{s}^{r} d t \int_{\mathbb{D}(t)}\left(\left(f_{[\nu]}\right)_{S_{[\nu]}}\right)^{*} \omega_{S_{[\nu]}}+\beta_{2}
$$

for $r \in(s, 1) \cap(\sigma+\delta / 4,+\infty)$. Here the constants $\beta_{1}>0, \beta_{2}>0$ are independent of the choice of $f \in \operatorname{Hol}(\mathbb{D}, X)$. By the induction hypothesis, we have

$$
\begin{aligned}
\min _{B \in \hat{\Lambda}}\left\{\int_{s}^{r} d t \int_{\mathbb{D}(t)}\left(\varpi_{B} \circ \hat{f}_{A}\right)^{*} \omega_{A / B}\right\} \leq & \frac{\varepsilon}{2} \int_{s}^{r} d t \int_{\mathbb{D}(t)} \hat{f}_{A}^{*} \omega_{A}+c_{1}^{\prime} \int_{s}^{r} d t \int_{\mathbb{D}(t)} \hat{f}_{\hat{S}}^{*} \omega_{\hat{S}} \\
& +c_{2}^{\prime} \max \left\{0, \int_{0}^{2 \pi} \log \frac{1}{\left|\hat{f}^{\prime}\left(s e^{i \theta}\right)\right|_{\omega_{A \times \hat{S}}}} \frac{d \theta}{2 \pi}\right\}+c_{3}^{\prime}
\end{aligned}
$$

holds for all $r \in(s, 1)$ outside some exceptional set whose linear measure is less than $\delta / 4$. Here the constants $c_{1}^{\prime}>0, c_{2}^{\prime}>0, c_{3}^{\prime}>0$ are independent of the choice of $f \in \operatorname{Hol}(\mathbb{D}, X)$. Hence combining the three estimates above, we get

$$
\begin{aligned}
\min _{B \in \Lambda}\left\{\int_{s}^{r} d t \int_{\mathbb{D}(t)}\left(\varpi_{B} \circ f_{A}\right)^{*} \omega_{A / B}\right\} & \leq \frac{\varepsilon}{2} \int_{s}^{r} d t \int_{\mathbb{D}(t)} f_{A}^{*} \omega_{A}+c_{1}^{\prime} \beta_{1} \int_{s}^{r} d t \int_{\mathbb{D}(t)}\left(\left(f_{[\nu]}\right)_{S_{[\nu]}}\right)^{*} \omega_{S_{[\nu]}} \\
+c_{2}^{\prime} \max & \left\{0, \int_{0}^{2 \pi} \log \frac{1}{\left|f^{\prime}\left(s e^{i \theta}\right)\right|_{\omega_{A \times S}}} \frac{d \theta}{2 \pi}\right\}+\left(c_{1}^{\prime} \beta_{2}+c_{3}^{\prime}\right)
\end{aligned}
$$

holds for all $r \in(\sigma, 1)$ outside some exceptional set whose linear measure is less than $\delta / 2$.

Now we combine two cases above. By (8.4) and (8.5), we get

$$
\begin{aligned}
\min _{B \in \Lambda}\left\{\int_{s}^{r} d t \int_{\mathbb{D}(t)}\left(\varpi_{B} \circ f_{A}\right)^{*} \omega_{A / B}\right\} & \leq \frac{\varepsilon}{2} \int_{s}^{r} d t \int_{\mathbb{D}(t)} f_{A}^{*} \omega_{A}+\left(4 \rho+c_{1}^{\prime} \beta_{1}\right) \int_{s}^{r} d t \int_{\mathbb{D}(t)}\left(f_{[\nu]}\right)_{S_{[\nu]}}^{*} \omega_{S_{[\nu]}} \\
& +c_{2}^{\prime} \max \left\{0, \int_{0}^{2 \pi} \log \frac{1}{\left|f^{\prime}\left(s e^{i \theta}\right)\right|_{\omega_{A \times S}}} \frac{d \theta}{2 \pi}\right\}+\left(c_{1}^{\prime} \beta_{2}+c_{3}^{\prime}\right)
\end{aligned}
$$

for all $r \in(s, 1)$ outside some exceptional set whose linear measure is less than $3 \delta / 4$. By Lemma 5.3, we have

$$
\begin{aligned}
\int_{s}^{r} d t \int_{\mathbb{D}(t)}\left(f_{[\nu]}\right)_{S_{[\nu]}}^{*} \omega_{S_{[\nu]}} \leq & \frac{\varepsilon}{2\left(4 \rho+c_{1}^{\prime} \beta_{1}\right)} \int_{s}^{r} d t \int_{\mathbb{D}(t)} f_{A}^{*} \omega_{A}+\mu_{1} \int_{s}^{r} d t \int_{\mathbb{D}(t)} f_{S}^{*} \omega_{S} \\
& +\mu_{2} \max \left\{0, \int_{0}^{2 \pi} \log \frac{1}{\left|f^{\prime}\left(s e^{i \theta}\right)\right|_{\omega_{A \times S}}} \frac{d \theta}{2 \pi}\right\}+\mu_{3}
\end{aligned}
$$


for all $r \in(s, 1)$ outside some exceptional set whose linear measure is less than $\delta / 4$. Here the constants $\mu_{1}>0, \mu_{2}>0, \mu_{3}>0$ are independent of the choice of $f \in \operatorname{Hol}(\mathbb{D}, X)$. Hence we get

$$
\begin{aligned}
\min _{B \in \Lambda}\left\{\int_{s}^{r} d t \int_{\mathbb{D}(t)}\left(\varpi_{B} \circ f_{A}\right)^{*} \omega_{A / B}\right\} \leq & \varepsilon \int_{s}^{r} d t \int_{\mathbb{D}(t)} f_{A}^{*} \omega_{A}+c_{1} \int_{s}^{r} d t \int_{\mathbb{D}(t)} f_{S}^{*} \omega_{S} \\
& +c_{2} \max \left\{0, \int_{0}^{2 \pi} \log \frac{1}{\left|f^{\prime}\left(s e^{i \theta}\right)\right|_{\omega_{A \times S}}} \frac{d \theta}{2 \pi}\right\}+c_{3}
\end{aligned}
$$

for all $r \in(s, 1)$ outside some exceptional set whose linear measure is less than $\delta$. Here we set

$$
c_{1}=\mu_{1}\left(4 \rho+c_{1}^{\prime} \beta_{1}\right), \quad c_{2}=c_{2}^{\prime}+\mu_{2}\left(4 \rho+c_{1}^{\prime} \beta_{1}\right), \quad c_{3}=\left(c_{1}^{\prime} \beta_{2}+c_{3}^{\prime}\right)+\mu_{3}\left(4 \rho+c_{1}^{\prime} \beta_{1}\right),
$$

which are positive constants independent of the choice of $f \in \operatorname{Hol}(\mathbb{D}, X)$. This conclude the induction step.

\section{Nevanlinna theory and blowing-ups: The case of abelian varieties.}

In Section 6, we discussed the estimate for $\hat{f}: \mathbb{D} \rightarrow \mathrm{Bl}_{Z} V$. When $V$ is an abelian variety, we obtain a better result than Lemma 6.1.

Lemma 9.1. Let $C$ be an abelian variety and let $Z \subset C$ be a closed subscheme such that the blowing-up $\mathrm{Bl}_{Z} C$ is smooth. Let $\omega_{C}$ be a positive invariant $(1,1)$-form on $C$ and let $\omega_{\mathrm{Bl}_{Z} C}$ be a smooth, positive (1,1)-form on $\mathrm{Bl}_{Z} C$. Let $\lambda_{Z}: C-\operatorname{supp} Z \rightarrow \mathbb{R}_{\geq 0}$ be a Weil function for $Z$. Let $W \subset C$ be an open neighborhood of $\operatorname{supp} Z$. Let $0<s<s^{\prime}<1$. Then there exist positive constants $\beta_{1}, \beta_{2}$ with the following property: Let $f \in \operatorname{Hol}(\mathbb{D}, C)$ be a holomorphic map such that $f(\mathbb{D}(s)) \not \subset W$. Then we have

$$
\int_{0}^{2 \pi} \lambda_{Z}\left(f\left(r e^{i \theta}\right)\right) \frac{d \theta}{2 \pi}+\int_{s}^{r} d t \int_{\mathbb{D}(t)} \hat{f}^{*} \omega_{\mathrm{Bl}_{Z} C} \leq \beta_{1} \int_{s}^{r} d t \int_{\mathbb{D}(t)} f^{*} \omega_{C}+\beta_{2}
$$

for $r \in\left[s^{\prime}, 1\right)$, where $\hat{f}: \mathbb{D} \rightarrow \mathrm{Bl}_{Z} C$ is the lift of $f$.

Proof of Lemma 9.1. We set $s^{\prime \prime}=\left(s+s^{\prime}\right) / 2$. We apply the two estimates (6.2) and (6.3) for $V=C$. We estimate the first term of the right hand side of (6.3). We prove that for each $f \in \operatorname{Hol}(\mathbb{D}, C)$ and each biholomorphic mapping $Q: \mathbb{D} \rightarrow \mathbb{D}\left(s^{\prime \prime}\right)$,

$$
\int_{0}^{1} \frac{d t}{t} \int_{\mathbb{D}(t)}(f \circ Q)^{*} \omega_{C} \leq \frac{1}{2\left(s^{\prime \prime}-s\right)} \int_{s}^{r} d t \int_{\mathbb{D}(t)} f^{*} \omega_{C}
$$

for $r \in\left(s^{\prime}, 1\right)$. To prove this, we set for $0 \leq t \leq 1$,

$$
\varphi(t)=\int_{0}^{2 \pi}\left|(f \circ Q)^{\prime}\left(t e^{i \theta}\right)\right|_{\omega_{C}}^{2} d \theta .
$$

Then since $\left|(f \circ Q)^{\prime}(z)\right|_{\omega_{C}}^{2}$ is subharmonic (cf. $\left.(2.1)\right), \varphi(t)$ is a positive increasing function. 
Hence we have

$$
\begin{aligned}
\int_{0}^{1} \frac{d t}{t} \int_{\mathbb{D}(t)}(f \circ Q)^{*} \omega_{C} & =\int_{0}^{1} \frac{d t}{t} \int_{0}^{t} \varphi(u) u d u=\int_{0}^{1} \frac{d t}{t}\left\{\left[\frac{u^{2}}{2} \varphi(u)\right]_{0}^{t}-\int_{0}^{t} \frac{u^{2}}{2} \varphi^{\prime}(u) d u\right\} \\
& \leq \int_{0}^{1} \frac{t^{2}}{2} \varphi(t) \frac{d t}{t}=\frac{1}{2} \int_{\mathbb{D}}(f \circ Q)^{*} \omega_{C}=\frac{1}{2} \int_{\mathbb{D}\left(s^{\prime \prime}\right)} f^{*} \omega_{C} .
\end{aligned}
$$

Since

$$
\int_{\mathbb{D}\left(s^{\prime \prime}\right)} f^{*} \omega_{C} \leq \frac{1}{s^{\prime}-s^{\prime \prime}} \int_{s^{\prime \prime}}^{s^{\prime}} d t \int_{\mathbb{D}(t)} f^{*} \omega_{C} \leq \frac{1}{s^{\prime \prime}-s} \int_{s}^{r} d t \int_{\mathbb{D}(t)} f^{*} \omega_{C}
$$

for $r \in\left(s^{\prime}, 1\right)$, we get $(9.1)$.

Now we complete the proof of Lemma 9.1. Set $\eta=\sup _{z \in C-W} \lambda_{Z}(z)$. Then by the assumption $f(\mathbb{D}(s)) \not \subset W$, we may take $w \in \mathbb{D}(s)$ such that $\lambda_{Z}(f(w)) \leq \eta$. Let $Q: \mathbb{D} \rightarrow \mathbb{D}\left(s^{\prime \prime}\right)$ be a biholomorphic mapping such that $Q(0)=w$. Then by $(6.2),(6.3)$ and (9.1), we have

$$
\begin{aligned}
& \int_{0}^{2 \pi} \lambda_{Z}\left(f\left(r e^{i \theta}\right)\right) \frac{d \theta}{2 \pi}+\int_{s}^{r} d t \int_{\mathbb{D}(t)} \hat{f}^{*} \omega_{\mathrm{Bl}_{Z} C} \\
& \quad \leq\left(\frac{\alpha_{1}}{s}+\frac{\alpha_{2} \alpha_{4}}{2\left(s^{\prime \prime}-s\right)^{2}}\right) \int_{s}^{r} d t \int_{\mathbb{D}(t)} f^{*} \omega_{C}+\alpha_{3}+\frac{\alpha_{2}}{s^{\prime \prime}-s}\left(\alpha_{5} \eta+\alpha_{6}\right)
\end{aligned}
$$

for $r \in\left(s^{\prime}, 1\right)$. The proof of Lemma 9.1 is finished.

\section{Proof of Theorem 2.1.}

We introduce the following lemma from which Theorem 2.1 follows.

Lemma 10.1. Let $B$ and $C$ be (possibly trivial) abelian varieties. Let $\omega_{B}$ and $\omega_{C}$ be positive invariant (1,1)-forms on $B$ and $C$, respectively. Let $Y \subset B \times C$ be a Zariski closed set. Let $U \subset Y$ be an open neighborhood of $\operatorname{Sp}(Y)$. Let $0<s<1, \delta>0$. Then there exist positive constants $\gamma_{1}, \gamma_{2}$ and $\gamma_{3}$ with the following property: For $f \in \operatorname{Hol}(\mathbb{D}, Y)$ with $f(\mathbb{D}(s)) \not \subset U$, we have

$$
\int_{s}^{r} d t \int_{\mathbb{D}(t)} f_{B}^{*} \omega_{B} \leq \gamma_{1} \int_{s}^{r} d t \int_{\mathbb{D}(t)} f_{C}^{*} \omega_{C}+\gamma_{2} \log ^{+} \frac{1}{\left|f^{\prime}(0)\right|_{\omega_{B \times C}}}+\gamma_{3}
$$

for all $r \in(s, 1)$ outside some exceptional set $E \subset(s, 1)$ whose linear measure is less than $\delta$.

Here $\operatorname{Sp}(Y)$ is the union of all positive dimensional translated abelian subvarieties of $B \times C$ which are contained in $Y$. Then $\operatorname{Sp}(Y)=\operatorname{Sp}\left(Y_{1}\right) \cup \cdots \cup \operatorname{Sp}\left(Y_{k}\right)$, where $Y_{1}, \ldots, Y_{k}$ are the irreducible components of $Y$. Hence $\operatorname{Sp}(Y)$ is a Zariski closed subset of $Y$.

We first derive Theorem 2.1 from Lemma 10.1. Let $X \subset A, \omega_{A}, U$ and $0<s<1$ be the objects described in Theorem 2.1. We apply Lemma 10.1 for the special case $B=A$, 
$C=\{0\}, Y=X$ and $\delta=(1-s) / 2$. Then, for $f \in \operatorname{Hol}(\mathbb{D}, X)$ with $f(\mathbb{D}(s)) \not \subset U$, we get

$$
\int_{s}^{(1+s) / 2} d t \int_{\mathbb{D}(t)} f^{*} \omega_{A} \leq \gamma_{2} \log ^{+} \frac{1}{\left|f^{\prime}(0)\right|_{\omega_{A}}}+\gamma_{3},
$$

and hence

$$
\int_{\mathbb{D}(s)} f^{*} \omega_{A} \leq \frac{2 \gamma_{2}}{1-s} \log ^{+} \frac{1}{\left|f^{\prime}(0)\right|_{\omega_{A}}}+\frac{2 \gamma_{3}}{1-s} .
$$

Since $\left|f^{\prime}(z)\right|_{\omega_{A}}^{2}$ is subharmonic on $\mathbb{D}($ cf. $(2.1))$, we have

$$
\left|f^{\prime}(0)\right|_{\omega_{A}}^{2} \leq \int_{0}^{2 \pi}\left|f^{\prime}\left(t e^{i \theta}\right)\right|_{\omega_{A}}^{2} \frac{d \theta}{2 \pi}
$$

Hence, we have

$$
\int_{\mathbb{D}(s)} f^{*} \omega_{A}=\int_{0}^{s} t d t \int_{0}^{2 \pi}\left|f^{\prime}\left(u e^{i \theta}\right)\right|_{\omega_{A}}^{2} d \theta \geq \pi s^{2}\left|f^{\prime}(0)\right|_{\omega_{A}}^{2} .
$$

Thus we have

$$
\left|f^{\prime}(0)\right|_{\omega_{A}} \leq \sqrt{\frac{2 \gamma_{2}}{\pi s^{2}(1-s)} \log ^{+} \frac{1}{\left|f^{\prime}(0)\right|_{\omega_{A}}}+\frac{2 \gamma_{3}}{\pi s^{2}(1-s)}}
$$

and hence

$$
\left|f^{\prime}(0)\right|_{\omega_{A}} \leq \max \left\{1, \sqrt{\frac{2 \gamma_{3}}{\pi s^{2}(1-s)}}\right\} .
$$

This conclude the derivation of Theorem 2.1 from Lemma 10.1.

Proof of Lemma 10.1. The proof is done by induction on $\operatorname{dim} B$. When $\operatorname{dim} B=0$, Lemma 10.1 is obvious. For the induction step, we assume that $\operatorname{dim} B>0$ and that Lemma 10.1 is true for all $B^{\prime} \in \Sigma(B)$. Let $p: Y \rightarrow C$ be the composite of the closed immersion $Y \hookrightarrow B \times C$ and the second projection $B \times C \rightarrow C$. We set $T=\left\{w \in C ; Y_{w}=B\right\}$. We may assume that $T \neq C$, for otherwise $Y=B \times C$, and hence our claim is trivial. We apply Lemma 3.1. There exists a closed subscheme $\mathcal{T} \subset C$ such that $\operatorname{supp} \mathcal{T}=T$ with the following property: If $\hat{Y} \subset B \times \mathrm{Bl}_{\mathcal{T}} C$ is the Zariski closure of $p^{-1}(C-T)$ in $Y \times_{C} \mathrm{Bl}_{\mathcal{T}} C \subset B \times \mathrm{Bl}_{\mathcal{T}} C$, then $\hat{Y}_{s} \neq B$ for all $s \in \mathrm{Bl}_{\mathcal{T}} C$. We may assume that $\mathrm{Bl}_{\mathcal{T}} C$ is a smooth projective variety equipped with a smooth positive $(1,1)$-form $\omega_{\mathrm{Bl}_{\mathcal{T}} C}$ (cf. Remark 3.2). We apply Proposition 2.3 for $\hat{Y} \subset B \times \mathrm{Bl}_{\mathcal{T}} C$ to get the non-empty, finite subset $\Lambda=\left\{B_{1}, \ldots, B_{n}\right\} \subset \Sigma(B)$.

For $B_{i} \in \Lambda$, let $\omega_{B_{i}}$ and $\omega_{B / B_{i}}$ be positive invariant $(1,1)$-forms on $B_{i}$ and $B / B_{i}$, respectively, and let $\varpi_{B_{i}}: B \rightarrow B / B_{i}$ be the quotient map. Assume that $f \in \operatorname{Hol}(\mathbb{D}, Y)$ satisfies $f(\mathbb{D}(s)) \not \subset U$. Using the induction hypothesis, we shall show that there exist positive constants $\rho_{i 1}, \rho_{i 2}, \rho_{i 3}$, which are independent of the choice of $f \in \operatorname{Hol}(\mathbb{D}, Y)$, such that 


$$
\begin{aligned}
\int_{s}^{r} d t \int_{\mathbb{D}(t)} f_{B}^{*} \omega_{B} \leq & \rho_{i 1}\left(\int_{s}^{r} d t \int_{\mathbb{D}(t)} f_{C}^{*} \omega_{C}+\int_{s}^{r} d t \int_{\mathbb{D}(t)}\left(\varpi_{B_{i}} \circ f_{B}\right)^{*} \omega_{B / B_{i}}\right) \\
& +\rho_{i 2} \log ^{+} \frac{1}{\left|f^{\prime}(0)\right|_{\omega_{B \times C}}}+\rho_{i 3}
\end{aligned}
$$

for all $r \in(s, 1)$ outside some exceptional set $E_{i}$ with the linear measure $\left|E_{i}\right|<\delta / 3 n$. We prove (10.1). We consider $B_{i}$ as an abelian subvariety $B_{i} \times\{0\} \subset B \times C$. We set $C_{i}=\left(B / B_{i}\right) \times C$. Then we have an exact sequence of abelian varieties

$$
0 \rightarrow B_{i} \rightarrow B \times C \rightarrow C_{i} \rightarrow 0
$$

By the Poincaré reducibility theorem, there exists an isogeny $\kappa: C_{i} \rightarrow C_{i}$ such that the pull back of the quotient map $B \times C \rightarrow C_{i}$ by $\kappa$ fits into the following commutative diagram:

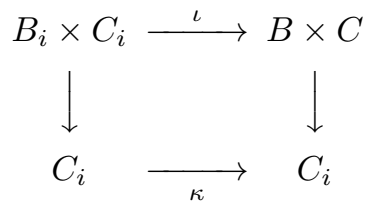

Here the map $B_{i} \times C_{i} \rightarrow C_{i}$ is the second projection and $\iota$ is an isogeny. Then $\operatorname{Sp}\left(\iota^{-1}(Y)\right)=\iota^{-1}(\operatorname{Sp}(Y))$. Hence $\operatorname{Sp}\left(\iota^{-1}(Y)\right) \subset \iota^{-1}(U)$. We denote by $\tilde{f} \in$ $\operatorname{Hol}\left(\mathbb{D}, \iota^{-1}(Y)\right)$ a lift of $f$. Then $\tilde{f}(\mathbb{D}(s)) \not \subset \iota^{-1}(U)$. Hence by the induction hypothesis, there exist positive constants $\gamma_{i 1}, \gamma_{i 2}, \gamma_{i 3}$ which are independent of the choice of $f \in \operatorname{Hol}(\mathbb{D}, Y)$ such that

$$
\int_{s}^{r} d t \int_{\mathbb{D}(t)} \tilde{f}_{B_{i}}^{*} \omega_{B_{i}} \leq \gamma_{i 1} \int_{s}^{r} d t \int_{\mathbb{D}(t)} \tilde{f}_{C_{i}}^{*} \omega_{C_{i}}+\gamma_{i 2} \log ^{+} \frac{1}{\left|\tilde{f}^{\prime}(0)\right|_{\omega_{B_{i} \times C_{i}}}}+\gamma_{i 3}
$$

for all $r \in(s, 1)$ outside some exceptional set $E_{i}$ with $\left|E_{i}\right|<\delta / 3 n$. Here $\omega_{C_{i}}=\omega_{\left(B / B_{i}\right) \times C}$ is the sum of the pull-bucks of $\omega_{B / B_{i}}$ and $\omega_{C}$. Hence we have

$$
\int_{s}^{r} d t \int_{\mathbb{D}(t)} \tilde{f}^{*} \omega_{B_{i} \times C_{i}} \leq\left(\gamma_{i 1}+1\right) \int_{s}^{r} d t \int_{\mathbb{D}(t)} \tilde{f}_{C_{i}}^{*} \omega_{C_{i}}+\gamma_{i 2} \log ^{+} \frac{1}{\left|\tilde{f}^{\prime}(0)\right|_{\omega_{B_{i} \times C_{i}}}}+\gamma_{i 3}
$$

for all $r \in(s, 1)$ outside $E_{i}$. There exists a positive constant $\mu_{i}>1$ such that

$$
\iota^{*} \omega_{B \times C} \leq \mu_{i} \omega_{B_{i} \times C_{i}} .
$$

Then we have

$$
\begin{aligned}
& \int_{s}^{r} d t \int_{\mathbb{D}(t)} f_{B}^{*} \omega_{B} \\
& \quad \leq \int_{s}^{r} d t \int_{\mathbb{D}(t)} f^{*} \omega_{B \times C} \leq \mu_{i} \int_{s}^{r} d t \int_{\mathbb{D}(t)} \tilde{f}^{*} \omega_{B_{i} \times C_{i}}
\end{aligned}
$$




$$
\begin{aligned}
& \leq \mu_{i}\left(\gamma_{i 1}+1\right) \int_{s}^{r} d t \int_{\mathbb{D}(t)} \tilde{f}_{C_{i}}^{*} \omega_{C_{i}}+\mu_{i} \gamma_{i 2} \log ^{+} \frac{1}{\left|\tilde{f}^{\prime}(0)\right|_{\omega_{B_{i} \times C_{i}}}}+\mu_{i} \gamma_{i 3} \\
& \leq \mu_{i}\left(\gamma_{i 1}+1\right) \int_{s}^{r} d t \int_{\mathbb{D}(t)} \tilde{f}_{C_{i}}^{*} \omega_{C_{i}}+\mu_{i} \gamma_{i 2} \log ^{+} \frac{1}{\left|f^{\prime}(0)\right|_{\omega_{B \times C}}}+\mu_{i} \gamma_{i 3}+\mu_{i} \gamma_{i 2} \frac{\log \mu_{i}}{2}
\end{aligned}
$$

for all $r \in(s, 1)$ outside $E_{i}$. There exists a positive constant $\mu_{i}^{\prime}$ such that

$$
\omega_{C_{i}} \leq \mu_{i}^{\prime} \kappa^{*} \omega_{C_{i}}
$$

Then we have

$$
\int_{s}^{r} d t \int_{\mathbb{D}(t)} \tilde{f}_{C_{i}}^{*} \omega_{C_{i}} \leq \mu_{i}^{\prime}\left(\int_{s}^{r} d t \int_{\mathbb{D}(t)} f_{C}^{*} \omega_{C}+\int_{s}^{r} d t \int_{\mathbb{D}(t)}\left(\varpi_{B_{i}} \circ f_{B}\right)^{*} \omega_{B / B_{i}}\right) .
$$

This shows (10.1). Here we set $\rho_{i 1}=\mu_{i} \mu_{i}^{\prime}\left(\gamma_{i 1}+1\right), \rho_{i 2}=\mu_{i} \gamma_{i 2}, \rho_{i 3}=\mu_{i} \gamma_{i 3}+$ $\mu_{i} \gamma_{i 2}\left(\left(\log \mu_{i}\right) / 2\right)$.

We set $\rho_{1}=\max _{1 \leq i \leq n}\left\{\rho_{i 1}\right\}, \rho_{2}=\max _{1 \leq i \leq n}\left\{\rho_{i 2}\right\}, \rho_{3}=\max _{1 \leq i \leq n}\left\{\rho_{i 3}\right\}$. Then by (10.1), we get

$$
\begin{aligned}
\int_{s}^{r} d t \int_{\mathbb{D}(t)} f_{B}^{*} \omega_{B} \leq & \rho_{1}\left(\int_{s}^{r} d t \int_{\mathbb{D}(t)} f_{C}^{*} \omega_{C}+\min _{1 \leq i \leq n} \int_{s}^{r} d t \int_{\mathbb{D}(t)}\left(\varpi_{B_{i}} \circ f_{B}\right)^{*} \omega_{B / B_{i}}\right) \\
& +\rho_{2} \log ^{+} \frac{1}{\left|f^{\prime}(0)\right|_{\omega_{B \times C}}}+\rho_{3}
\end{aligned}
$$

for all $r \in(s, 1)$ outside $E^{\prime}$, where $E^{\prime}=\bigcup_{1 \leq i \leq n} E_{i}$. Then $\left|E^{\prime}\right|<\delta / 3$.

Now we take $\varepsilon>0$ so that $\varepsilon \rho_{1}<1 / 2$. By $p^{-1}(T) \subset \operatorname{Sp}(Y)$ and $f(\mathbb{D}(s)) \not \subset U$, we have $f_{C}(\mathbb{D}) \not \subset T$. Hence there exists a unique lifting $\hat{f} \in \operatorname{Hol}(\mathbb{D}, \hat{Y})$ of $f \in \operatorname{Hol}(\mathbb{D}, Y)$. By Proposition 2.3, there exist positive constants $c_{1}^{\prime}, c_{2}^{\prime}$ and $c_{3}^{\prime}$, which are independent of the choice of $f \in \operatorname{Hol}(\mathbb{D}, Y)$, such that

$$
\begin{aligned}
\min _{1 \leq i \leq n} & \left\{\int_{s}^{r} d t \int_{\mathbb{D}(t)}\left(\varpi_{B_{i}} \circ f_{B}\right)^{*} \omega_{B / B_{i}}\right\} \\
\leq & \varepsilon \int_{s}^{r} d t \int_{\mathbb{D}(t)} f_{B}^{*} \omega_{B}+c_{1}^{\prime} \int_{s}^{r} d t \int_{\mathbb{D}(t)}\left(\hat{f}_{\mathrm{Bl}_{\mathcal{T}} C}\right)^{*} \omega_{\mathrm{Bl} \mathcal{T} C} \\
& +c_{2}^{\prime} \max \left\{0, \int_{0}^{2 \pi} \log \frac{1}{\left|\hat{f}^{\prime}\left(s e^{i \theta}\right)\right|_{\omega_{B \times \mathrm{Bl}_{\mathcal{T}} C}}} \frac{d \theta}{2 \pi}\right\}+c_{3}^{\prime}
\end{aligned}
$$

for all $r \in(s, 1)$ outside some exceptional set $E^{\prime \prime}$ with $\left|E^{\prime \prime}\right|<\delta / 3$. We take a positive constant $\mu^{\prime \prime}>1$ such that $\alpha^{*} \omega_{B \times C} \leq \mu^{\prime \prime} \omega_{B \times \mathrm{Bl}_{\mathcal{T}} C}$ where $\alpha: B \times \mathrm{Bl}_{\mathcal{T}} C \rightarrow B \times C$ is the induced map. Then we have

$$
\int_{0}^{2 \pi} \log \frac{1}{\left|\hat{f}^{\prime}\left(s e^{i \theta}\right)\right|_{\omega_{B \times \mathrm{Bl}} \mathcal{T}^{C}}} \frac{d \theta}{2 \pi} \leq \int_{0}^{2 \pi} \log \frac{1}{\left|f^{\prime}\left(s e^{i \theta}\right)\right|_{\omega_{B \times C}}} \frac{d \theta}{2 \pi}+\frac{\log \mu^{\prime \prime}}{2} .
$$

Since $\log \left|f^{\prime}\right|_{\omega_{B \times C}}$ is subharmonic on $\mathbb{D}($ cf. (2.1)), we have 


$$
\int_{0}^{2 \pi} \log \frac{1}{\left|f^{\prime}\left(s e^{i \theta}\right)\right|_{\omega_{B \times C}}} \frac{d \theta}{2 \pi} \leq \log \frac{1}{\left|f^{\prime}(0)\right|_{\omega_{B \times C}}} \leq \log ^{+} \frac{1}{\left|f^{\prime}(0)\right|_{\omega_{B \times C}}} .
$$

Hence we get

$$
\begin{aligned}
\min _{1 \leq i \leq n} & \left\{\int_{s}^{r} d t \int_{\mathbb{D}(t)}\left(\varpi_{B_{i}} \circ f_{B}\right)^{*} \omega_{B / B_{i}}\right\} \\
\leq \varepsilon & \int_{s}^{r} d t \int_{\mathbb{D}(t)} f_{B}^{*} \omega_{B}+c_{1}^{\prime} \int_{s}^{r} d t \int_{\mathbb{D}(t)}\left(\hat{f}_{\mathrm{Bl}_{\mathcal{T}} C}\right)^{*} \omega_{\mathrm{Bl} \mathcal{T} C}+c_{2}^{\prime} \log ^{+} \frac{1}{\left|f^{\prime}(0)\right|_{\omega_{B \times C}}}+c_{3}^{\prime \prime}
\end{aligned}
$$

for all $r \in(s, 1)$ outside $E^{\prime \prime}$, where we set $c_{3}^{\prime \prime}=c_{2}^{\prime}\left(\left(\log \mu^{\prime \prime}\right) / 2\right)+c_{3}^{\prime}$. We combine this estimate with (10.2) to get

$$
\begin{aligned}
\int_{s}^{r} d t \int_{\mathbb{D}(t)} f_{B}^{*} \omega_{B} \leq & 2 \rho_{1} \int_{s}^{r} d t \int_{\mathbb{D}(t)} f_{C}^{*} \omega_{C}+2 c_{1}^{\prime} \rho_{1} \int_{s}^{r} d t \int_{\mathbb{D}(t)}\left(\hat{f}_{\mathrm{Bl}_{\mathcal{T}} C}\right)^{*} \omega_{\mathrm{Bl}{ }_{\mathcal{T}} C} \\
& +2\left(c_{2}^{\prime} \rho_{1}+\rho_{2}\right) \log ^{+} \frac{1}{\left|f^{\prime}(0)\right|_{\omega_{B \times C}}}+2\left(c_{3}^{\prime \prime} \rho_{1}+\rho_{3}\right)
\end{aligned}
$$

for all $r \in(s, 1)$ outside $E^{\prime} \cup E^{\prime \prime}$, where $\left|E^{\prime} \cup E^{\prime \prime}\right|<2 \delta / 3$.

Now we apply Lemma 9.1 to estimate the right hand side. We have $p^{-1}(T) \subset \operatorname{Sp}(Y)$, and hence $p^{-1}(T) \subset U$. Thus there exists an open neighborhood $W \subset C$ of $T$ such that $p^{-1}(W) \subset U$. Then by $f(\mathbb{D}(s)) \not \subset U$, we have $f_{C}(\mathbb{D}(s)) \not \subset W$. Hence, by Lemma 9.1 , there exist positive constants $\beta_{1}$ and $\beta_{2}$, which are independent of the choice of $f \in \operatorname{Hol}(\mathbb{D}, Y)$, such that

$$
\int_{s}^{r} d t \int_{\mathbb{D}(t)}\left(\hat{f}_{\mathrm{Bl}_{\mathcal{T}} C}\right)^{*} \omega_{\mathrm{Bl}_{\mathcal{T}} C} \leq \beta_{1} \int_{s}^{r} d t \int_{\mathbb{D}(t)} f_{C}^{*} \omega_{C}+\beta_{2}
$$

for $r \in(s, 1)$ outside $(s, s+\delta / 3)$. Combining this estimate with (10.3), we get

$$
\begin{aligned}
& \int_{s}^{r} d t \int_{\mathbb{D}(t)} f_{B}^{*} \omega_{B} \\
& \leq 2 \rho_{1}\left(1+c_{1}^{\prime} \beta_{1}\right) \int_{s}^{r} d t \int_{\mathbb{D}(t)} f_{C}^{*} \omega_{C}+2\left(c_{2}^{\prime} \rho_{1}+\rho_{2}\right) \log ^{+} \frac{1}{\left|f^{\prime}(0)\right|_{\omega_{B \times C}}}+2\left(c_{3}^{\prime \prime} \rho_{1}+\rho_{3}+c_{1}^{\prime} \rho_{1} \beta_{2}\right)
\end{aligned}
$$

for $r \in(s, 1)$ outside $E$, where $E=E^{\prime} \cup E^{\prime \prime} \cup(s, s+\delta / 3)$. Then $|E|<\delta$. This establishes the estimate of Lemma 10.1 for $B$, where

$$
\gamma_{1}=2 \rho_{1}\left(1+c_{1}^{\prime} \beta_{1}\right), \quad \gamma_{2}=2\left(c_{2}^{\prime} \rho_{1}+\rho_{2}\right), \quad \gamma_{3}=2\left(c_{3}^{\prime \prime} \rho_{1}+\rho_{3}+c_{1}^{\prime} \rho_{1} \beta_{2}\right) .
$$

Note that these are positive constants which are independent of the choice of $f \in$ $\operatorname{Hol}(\mathbb{D}, Y)$. This completes the proof of Lemma 10.1 . 


\section{Complex subspaces of complex tori.}

So far, we treat the case of subvarieties of abelian varieties. However, our results can be generalized to the case of complex subspaces of complex tori by the following lemma.

LEMma 11.1. Let $X$ be a closed complex subspace of a complex torus T. Assume that $X$ is of general type. Then there exists a complex subtorus $A \subset T$ which is an abelian variety such that $X$ is contained in some translate of $A$. In particular, $X$ is a closed subvariety of an abelian variety.

Proof. This lemma follows from [32, Lemma 10.8]. We give a proof for completeness. Since $X$ is of general type, $X$ is a Moishezon space. Hence by a theorem of Moishezon, there exists a bimeromorphic modification $\hat{X} \rightarrow X$ such that $\hat{X}$ is smooth and projective (cf. [32, Theorem 3.6]). We consider the Albanese map $\alpha: \hat{X} \rightarrow \operatorname{Alb}(\hat{X})$. Then $\operatorname{Alb}(\hat{X})$ is an abelian variety. Let $\hat{\iota}: \hat{X} \rightarrow T$ be the composite of $\hat{X} \rightarrow X$ and the immersion $\iota: X \hookrightarrow T$. By the universal property of the Albanese map, there exists a holomorphic map $h: \operatorname{Alb}(\hat{X}) \rightarrow T$ such that $h \circ \alpha=\hat{\imath}$. The image $h(\operatorname{Alb}(\hat{X}))$ is a translate of a complex subtorus $A \subset T$. Then $A$ is an abelian variety, for $A$ is a quotient of the abelian variety $\operatorname{Alb}(\hat{X})$. We have $X=\hat{\imath}(\hat{X}) \subset h(\operatorname{Alb}(\hat{X}))$.

Combining Lemma 11.1 with Corollary 1.1, we immediately obtain the following

Corollary 11.2. Let $X$ be a closed complex subspace of a complex torus. Assume that $X$ is of general type. Then $X$ is pseudo Kobayashi hyperbolic.

Let $X$ be a closed complex subspace of a complex torus $T$. Then we may define the special set $\operatorname{Sp}(X)$ by

$$
\operatorname{Sp}(X)=\left\{x \in X ; \exists T^{\prime} \subset T \text {, a complex subtorus s.t. } \operatorname{dim}\left(T^{\prime}\right)>0 \text { and } x+T^{\prime} \subset X\right\} .
$$

If $X$ is not of general type, then by [32, Theorem 10.9], we have $\operatorname{Sp}(X)=X$. If $X$ is of general type, then by Lemma 11.1, there exists an abelian variety $A$ such that $X \subset A$. The special set of $X$ which is defined as a subvariety of the abelian variety $A$ is equal to our special set $\operatorname{Sp}(X)$. Hence by Theorem 1.4, we obtain the following

Corollary 11.3. Let $X$ be a closed complex subspace of a complex torus $T$. Then $X$ is taut modulo $\operatorname{Sp}(X)$. In particular, $X$ is Kobayashi hyperbolic modulo $\operatorname{Sp}(X)$.

Next we prove the following

Corollary 11.4. Let $X$ be a closed complex subspace of a complex torus T. Assume that there exists a subset $E \subset X$ such that

- $d_{X}(x, y)=0$ for all $x, y \in E$, and

- $E$ is not contained in any proper analytic subset of $X$.

Then $X$ is a translate of a complex subtorus of $T$. 
Proof. Let $T_{0} \subset T$ be the maximal complex subtorus which stabilizes $X$. Let $W \subset T / T_{0}$ be the image of $X$ under the quotient $T \rightarrow T / T_{0}$. We show $\operatorname{dim} W=0$. Assume contrary that $\operatorname{dim} W>0$. By [32, Theorem 10.9] or [19, Corollary 3.8.28], $W$ is of general type. Hence by Corollary 11.2, $W$ is pseudo Kobayashi hyperbolic, i.e., there exists a proper Zariski closed set $Z \varsubsetneqq W$ such that $W$ is Kobayashi hyperbolic modulo $Z$. Let $\varphi: X \rightarrow W$ be the induced morphism. Since $\varphi$ is surjective, $\varphi(E)$ is Zariski dense in $W$. By the distance decreasing property of Kobayashi pseudo distances, $d_{W}(p, q)=0$ for all $p, q \in \varphi(E)$. By $\operatorname{dim} W>0$, we may take distinct points $p, q \in \varphi(E) \backslash Z$. This is a contradiction. Thus $\operatorname{dim} W=0$. Hence $X$ is a translate of a complex subtorus of $T$.

Finally, we prove the following corollary from which Corollary 1.5 immediately follows.

Corollary 11.5. Let $X$ be a compact complex manifold. Assume that there exists a subset $E \subset X$ such that

- $d_{X}(x, y)=0$ for all $x, y \in E$, and

- $E$ is not contained in any proper analytic subset of $X$.

Then the Albanese map $\alpha: X \rightarrow \operatorname{Alb}(X)$ is surjective. If moreover $X$ is Kähler, then we have $q(X) \leq \operatorname{dim}(X)$.

As a consequence, if $X$ is a compact complex manifold such that $d_{X} \equiv 0$, then the Albanese map $\alpha: X \rightarrow \operatorname{Alb}(X)$ is surjective. For a related discussion of this statement in the context of Campana's theory of special varieties, we refer the readers to [4, Section 9.3].

Proof of Corollary 11.5. Set $Y=\alpha(X)$. Then $Y$ is a closed complex subspace of $\operatorname{Alb}(X)$. The image $\alpha(E) \subset Y$ is not contained in any proper analytic subset of $Y$. By the distance decreasing property of Kobayashi pseudo distances, we have $d_{Y}(p, q)=0$ for all $p, q \in \alpha(E)$. Thus by Corollary 11.4, $Y$ is a translate of a complex subtorus of $\operatorname{Alb}(X)$. By the universal property of the Albanese map, we have $Y=\operatorname{Alb}(X)$. Hence the Albanese map $\alpha: X \rightarrow \operatorname{Alb}(X)$ is surjective (cf. [32, Corollary 10.6]). If moreover $X$ is Kähler, then we have $\operatorname{dim}(\operatorname{Alb}(X))=q(X)$. Thus $q(X) \leq \operatorname{dim}(X)$. This shows Corollary 11.5.

Acknowledgments. The author would like to thank the referee and the editors for their valuable comments which helped to improve the presentation of the paper.

\section{References}

[1] A. Bloch, Sur les systèmes de fonctions holomorphes à variétés linéaires lacunaires, Ann. École Normale, 43 (1926), 309-362.

[ 2 ] A. Bloch, Sur les systèmes de fonctions uniformes satisfaisant à l'équation d'une variété algébrique dont l'irrégularité dépasse la dimension, J. Math. Pures Appl., 5 (1926), 19-66.

[3] M. Brunella, Courbes entières dans les surfaces algébriques complexes, Astérisque, 282 (2002), 39-61. 
[4] F. Campana, Orbifolds, special varieties and classification theory, Ann. Inst. Fourier (Grenoble), 54 (2004), 499-630.

[ 5 ] H. Cartan, Sur les systèmes de fonctions holomorphes à variétés linéaires lacunaires et leurs applications, Ann. École Normale, 45 (1928), 255-346.

[6] J. P. Demailly, Algebraic criteria for Kobayashi hyperbolic projective varieties and jet differentials, Proc. of Sympos. in Pure Math., 62, American Mathematical Society, 1997, 285-360.

[ 7 ] B. Edixhoven and J. H. Evertse (Eds.), Diophantine Approximation and Abelian Varieties, Lecture Notes in Math., 1566, Springer, 1993.

[ 8] L. C. Evans and R. F. Gariepy, Measure theory and fine properties of functions, CRC press, 1991.

[9] M. Green, Holomorphic maps to complex tori, Amer. J. Math., 100 (1978), 615-620.

[10] M. Green and P. Griffiths, Two applications of algebraic geometry to entire holomorphic mappings, The Chern symposium, 1979, Springer, New York, 1980.

[11] R. Hartshorne, Connectedness of the Hilbert scheme, Publ. Math. IHÉS, 29 (1966), 5-48.

[12] R. Hartshorne, Algebraic geometry, Graduate Texts in Math., 156, Springer-Verlag, Berlin, 1977.

[13] W. K. Hayman, Subharmonic functions Vol. 2, London Math. Soc. Monographs, 20, Academic Press, Inc., London, 1989.

[14] H. Hironaka, Flattening theorem in complex-analytic geometry, Amer. J. Math., 97 (1975), 503547.

[15] Y. Kawamata, On Bloch's conjecture, Invent. Math., 57 (1980), 97-100.

[16] P. Kiernan and S. Kobayashi, Holomorphic mappings into projective space with lacunary hyperplanes, Nagoya Math. J., 50 (1973), 199-216.

[17] R. Kobayashi, Holomorphic curves into algebraic subvarieties of an abelian variety, Internat. J. Math., 2 (1991), 711-724.

[18] R. Kobayashi, Nevanlinna theory and number theory (Japanese), Sūgaku, 48 (1996), 113-127.

[19] S. Kobayashi, Hyperbolic Complex Spaces, Springer, 1998.

[20] S. Lang, Introduction to Complex Hyperbolic Spaces, Springer-Verlag, 1987.

[21] S. Lang, Survey of Diophantine Geometry, Springer-Verlag, 1997.

[22] M. McQuillan, A new proof of the Bloch conjecture, J. Algebraic Geometry, 5 (1996), 107-118.

[23] M. McQuillan, Diophantine approximations and foliations, Publ. Math. I.H.E.S., 87 (1998), 121174 .

[24] D. Mumford, Abelian varieties, Oxford University Press, 1974.

[25] J. Noguchi, Lemma on logarithmic derivatives and holomorphic curves in algebraic varieties, Nagoya Math. J., 83 (1981), 213-233.

[26] J. Noguchi and T. Ochiai, Geometric function theory in several complex variables, Transl. Math. Mon., 80, Amer. Math. Soc., Providence, R. I., 1990.

[27] J. Noguchi and J. Winkelmann, Holomorphic curves and integral points off divisors, Math. Z., 239 (2002), 593-610.

[28] J. Noguchi and J. Winkelmann, Nevanlinna Theory in Several Complex Variables and Diophantine Approximation, Springer, 2013.

[29] T. Ochiai, On holomorphic curves in algebraic varieties with ample irregularity, Invent. Math., 43 (1977), 83-96.

[30] M. Raynaud, Géométrie algébrique et géométrie analytique, Lecture Notes in Math., 224, Springer, 1971, 311-343.

[31] E. Sernesi, Deformations of algebraic schemes, Grundlehren der Mathematischen Wissenschaften, 334, Springer-Verlag, 2006.

[32] K. Ueno, Classification theory of algebraic varieties and compact complex spaces, Lecture Notes in Math., 439, Springer-Verlag, 1975.

[33] P. Vojta, On the ABC conjecture and Diophantine approximation by rational points, Amer. J. Math., 122 (2000), 843-872.

[34] J. Winkelmann, On Brody and entire curves, Bull. Soc. Math. France, 135 (2007), 25-46.

[35] K. Yamanoi, Algebro-geometric version of Nevanlinna's lemma on logarithmic derivative and applications, Nagoya Math. J., 173 (2004), 23-63. 


\section{Katsutoshi YAmanoI}

Department of Mathematics

Graduate School of Science

Osaka University

Toyonaka

Osaka 560-0043, Japan

E-mail: yamanoi@math.sci.osaka-u.ac.jp 